\title{
The nature and context of Kaaps: a contemporary, past and future perspective
}

\author{
Frank Hendricks \\ University of the Western Cape
}

\begin{abstract}
In this contribution, which serves as orientation for this special edition, the accent falls chiefly on the contemporary manifestation of Kaaps as colloquial variety of Afrikaans, but also on its historic roots and the challenges regarding its future. Besides a reflection on the name "Kaaps" and other alternative names, this language form is described with reference to its origin, traditional speakers, geographic situation, sociolectic nature, linguistic character and its contextual usages. Kaaps is presented as a variety of the dialect group Southwestern Afrikaans which as a form of colloquial Afrikaans refers back historically to the seventeenth century influence of slaves on the formation of Afrikaans and which is currently chiefly manifested as a sociolect associated with the working class of the Cape Peninsula. The social assessment, lingua-political treatment and survival potential of this variety are also reviewed.
\end{abstract}

\section{INTRODUGTION ${ }^{1}$}

The language form Kaaps is reflected in utterances like the following, as well as the example materials in in the section 'THE LINGUISTIC NATURE OF KAAPS'::

- Aweh, Vader Krismis. Bly om te sien djy's nog altyd soe mal oor cowboys en kroeks speel. [Hi, Father Christmas. Glad to see you're still so crazy about playing cowboys and crooks.] (Son, 12/11/2012)

- Ma' nou wat djy op varsity is, dink djy djy's slimmere as my. [But now that you are at university, you think you are cleverer than me.] (Zulfah Otto-Sallies, Diekie vannie Bo-Kaap, p. 39)

- Wel - hoe s'l 'k nou sê - ons het oek maa' onse aps-en-douns; net soes elke-iene: vandag is die blomme baieng skaa's, môre kry djy wee' glad te veel... [Well - how should I put it we also have our ups and downs; just like everyone: today the flowers are scarce, tomorrow you have far too many...] (S.V. Petersen, 'In die hart van die stad')

\footnotetext{
1 This paper is an adapted version of the opening paper I delivered during the symposium on Kaaps at the University of the Western Cape from 19 to 20 July 2012. The title of the paper was 'The nature and context of Kaaps: a past, contemporary and future perspective'.
} 
- Djy moet daai mond van jou control. Is bêd vir business. Djy wiet vir jouself times is bêd...! [You must control that mouth of yours. It's bad for business. You know yourself times are bad...!] (Peter Snyders, Political Joke, p. 9)

This language form has its roots in the Cape Peninsula, is still virile, and is chiefly linked, as will be shown later, with the working class of this area.

This orienting contribution takes a closer look at the nature and contextual aspects of Kaaps. My approach is linguistic, more specifically variationallinguistic. Accordingly, I consider languages (Afrikaans, English, isiXhosa, Dutch, etc.) to be entities which are each made up of a network of varieties. Theoretically, I favour an egalitarian approach to language diversity. This approach rests on the assumption that the standard variety and colloquial varieties of a language (such as Afrikaans) are intertwined organically (Ponelis 1994:117) and should be regarded as entities on the same level, thus as linguistically equivalent and equally valid codes of communication (Hendricks 2012a:51, 2012b:96). My perspective (of the topic) is, as indicated in the title, chiefly contemporary but also historically and future oriented. Born and bred in the Hantam, I would not be able to pass for a primary speaker of Kaaps, but I can speak and write it in a way. After all, I have been exposed to Kaaps since my childhood - and am thus able to judge the linguistic aspects and use thereof (whether verbal or written) from within.
Organisationally, this contribution comprises the following: firstly, the dinstinctive nature of Kaaps is described in terms of its naming, its varying linguistic nature and its social aspects. Thereafter the emphasis falls on its social assessment and lingua-political handling. In closing, the future of Kaaps is touched on with reference to some relevant challenges. Regarding its functional tenor, my approach seeks to be informative, but also speculative and direction-seeking.

\section{THE NAME KAAPS AND ALTERNATIVE NAMES}

The name 'Kaaps' which is currently used generally and in academic circles for this language form, correctly implies that this is a language form which was formed in Cape Town and environs - and not elsewhere. Adam Small played a leading role in settling on the name 'Kaaps' for this language form (Gerwel 2012:17). Customary alternatives for 'Kaaps' are, firstly, 'Kaapse Vernakulêre Afrikaans' (Ponelis 2009)', or 'Kaapse VernakulêrAfrikaans' ['Cape Vernacular-Afrikaans'] (Du Plessis 1987:130), and, secondly, 'Kaapse Afrikaans' ["Cape Afrikaans"] (Hendricks 1978:13-26; Carstens 2003:291; Le Cordeur 2011:763-766).

The alternative name 'Kaapse Vernakulêre Afrikaans' ['Cape Vernacular Afrikaans'] rightly indicates that Kaaps is by nature a form of Vernacular Afrikaans ${ }^{3}$ (a countrywide phenomenon) as manifested in Cape Town and environs. Vernacular Afrikaans is, according to Ponelis (1998:5), a distinctive form of colloquial Afrikaans, which, inter alia,

2 The English equivalents for "Kaapse Vernakulêre Afrikaans", used by Ponelis, are "Cape Vernacular Afrikaans" (Ponelis 1996:136) and "the vernacular Afrikaans of Cape Town" (Ponelis 1996:131).

3 Ponelis (1998:5) makes use of the alternative spelling "Vernekulêre Afrikaans". 
(a) has a hyper-informal slant, (b) is deeply under the influence of English, (c) is subject to relatively rapid renewal and change, and (d) is spoken especially by people from the lower social class but also by people from the middle and upper class. In a later work (Ponelis 2009) Ponelis uses the term 'demotiese Afrikaans' ['demotic Afrikaans'] as alternative name for 'Vernacular Afrikaans'. He also states categorically that demotic Afrikaans occurs nationally and that Cape Vernacular Afrikaans is only one of its manifestation forms:

The users of demotic Afrikaans occur throughout the Afrikaans language community, in all regions, in all social groups, from barely literate to learned bigwigs. This thus means that Cape Vernacular Afrikaans is only one form of demotic Afrikaans, but not the only one by far.

The use of 'Kaapse Afrikaans' ['Cape Afrikaans'], as alternative for 'Kaaps', could create confusion, as in some works on Afrikaans language variation (Ponelis 1987:9, 1989a:13; Botha 1989:127) the term 'Kaapse Afrikaans' is used synonymously with the term 'Suidwestelike Afrikaans' ['Southwestern Afrikaans'] in order to refer to the colloquial Afrikaans spoken in that area of South Africa, known earlier as 'Suidwes-Kaapland' (the Southwestern Cape), and currently as 'die Wes-Kaap' ['the Western Cape']. As such, the name 'Cape Afrikaans' covers besides Kaaps (as chiefly working class language form typical of the Cape Peninsula) also other colloquial distinctions in the Western Cape such as Boland, Swartland and Overberg Afrikaans (Ponelis 1987:9, 1998:15). ${ }^{4}$

It is indeed interesting to note that 'Kaaps' is no recent name. Nienaber (1952:63-65) points out that during the Dutch and early English rule at the Cape, specifically in the period before the Great Trek and the establishment of the Boer republics, 'Kaaps' was used locally as well as by foreigners as a shortened name for 'Kaaps-Hollands' ['Cape Dutch'] when referring to the South African form of Dutch, used at the Cape in the seventeenth century and thereafter. It also turned up as component in a number of alternatives for 'KaapsHollands', including 'Kaapsch Taaleigen' [own Cape language] and 'Kaapschen tongval' ['Cape tongue/dialect']. These earlier names seem to correspond conceptually and/or regarding range value with the terms 'Cape Afrikaans' and 'South western Afrikaans' (as used by Ponelis). Kaaps (as primarily working class variety) is thus linked by name with Cape-Dutch as earlier layer of the Afrikaans language.

\section{THE VARIATIONAL- LINGUISTIC VIEW OF KAAPS}

From a variational-linguistic perspective, i.e. dialectically and socio-linguistically, Kaaps is relatively nuanced. ${ }^{5}$ This nuanced character will subsequently be

4 For Van Rensburg 'Cape Afrikaans' has the same range value as 'South western Afrikaans' because he (Van Rensburg 1989:439) explicates that the three dialect collections of Ponelis (1987:9), i.e. South western, North western and Eastern Afrikaans, match his own trichotomy: 'Cape Afrikaans', 'Orange River Afrikaans' and 'Eastern Border Afrikaans'. His elucidation of 'Cape Afrikaans', using only examples from the erstwhile Slave Afrikaans and the contemporary colloquial Afrikaans of Coloured and/or Muslim speakers in the Cape Peninsula (Van Rensburg 1989:449-451), may however create the impression that the term 'Cape Afrikaans' is only applicable to the latter.

5 Carstens (2003:291) states in this regard, 'It is difficult to exactly define "Cape Afrikaans". It is mainly limited geographically to the Cape environs (thus a geolect), but it also shows characterics of social stratification (thus a sociolect).' 
elucidated with reference to the linguistic status, early traces, geolectic nature and the speaker corps of this language form.

\section{The linguistic status of Kaaps}

Regarding its linguistic status, Kaaps should be regarded as a variety of Afrikaans - not as a language or language form alongside Afrikaans. The alternative name 'Cape Afrikaans' implicitly alludes to the fact that Kaaps is essentially a form of Afrikaans.

Furthermore Kaaps is, as is clear from the component 'vernacular' in the aforementioned alternative names 'Cape Vernacular Afrikaans' (Ponelis 2009) ${ }^{6}$ and 'Cape Vernacular-Afrikaans' (Du Plessis 1987:130), more specifically a colloquial variety of Afrikaans, thus a form of spoken Afrikaans. As such, Kaaps, together with all other distinguishable colloquial varieties of Afrikaans, is organically linked to, and thus in interaction with, Standard Afrikaans as commonly used unitary variety.

\section{The early traces of Kaaps}

Kaaps is one of the oldest varieties of Afrikaans, and its development coincides with that of Afrikaans as a language at large. In terms of its historic origin, contemporary Kaaps can be traced back to the following:

a) Europe, Africa and the East, specifically the seventeenth century Afrikaansification of Dutch at the Cape under the influence of slaves from Asia (Malaysia, Java, Indonesia, India), Madagascar and Mozambique, who, besides their own mother tongues, spoke Pasar Malay and Creole Portuguese (Van
Rensburg 1989:463 ff., Van Rensburg 1997:10 ff.; Kotzé 2001:108) as well as

b) the impact of English influence after circa 1806.

Accordingly, Kaaps can be regarded, historically speaking, as a linguistic offshoot of two consecutive periods of colonial rule at the Cape, i.e. the Dutch or VOC period until about the end of the 18th century, and English rule from the beginning of the 19th century. In this regard, Ponelis (1987:5-7; 1989a:11; 1999a) points out that during the formative phase of Afrikaans it was especially the influence of Malay and Low Portuguese (the dominant languages of slave communication) which caused the transformation of Dutch to AfrikaansDutch, through language influence (borrowing) and language interference.

These early traces of Kaaps can be associated with contemporary sociolinguistic research, which focuses on the origin and use of so-called 'supervernaculars' as reflection of 'superdiversity', i.e. a social phenomenon that has manifested in cities like Antwerp and Cape Town since the 1990s. Accordingly, one could argue retrospectively that Kaaps took root in the period of Dutch rule at the Cape and the early years of English rule, and that it was utilised as a sort of supervernacular within a social context, specifically a slave context, which could be typified as superdiverse, or as multinational and multicultural.

\section{The geolectic nature/geographic situation of Kaaps}

As indicated earlier, Kaaps is identified geolectially as a subvariety of the dialect bundle Southwestern Afrikaans (Ponelis

6 In contrast to Ponelis, Müller and Pistor (2011:36) prefer the orthographic form 'Cape vernacular Afrikaans' (thus 'vernacular' in lowercase). 
1987:9). As such, Kaaps has taken shape historically in the proximity of Swartland, Boland, Overberg and West Coast Afrikaans, among others. In this regard, Ponelis points out that Afrikaans diversified into three dialects in the eighteenth century, namely Southwestern, Northwestern and Eastern Afrikaans (Ponelis 1994; 1998:3) and that slaves who spoke Malay and Low Portuguese had a strong influence on Southwestern Afrikaans during this regionalisation phase (Ponelis 1996:130). Ponelis (1996:131) further indicates that language phenomena, currently considered typical of Cape Vernacular Afrikaans, were also prevalent in other areas where Southwestern Afrikaans was spoken, but eventually fell out of use under the pressure of the standard variety. As such, the contemporary Cape working class language form can be seen as a distinct variety in which the original linguistic character of the Southwestern Afrikaans dialect has been retained to a significant extent.

Moving closer geographically, Kaaps is chiefly limited to the Cape Peninsula, with the following areas as likely places of origin (Hendricks 1978:20):

a) the Bo-Kaap (the historic centre of the Cape Muslim culture since the late $1800 \mathrm{~s}$ and which since about 1836 was inhabited by freed slaves (Anon n.d.:236)) and

b) District Six (founded in 1867 and a cosmopolitan residential area inhabited by freed slaves, workers, immigrants, traders and artists (Anon n.d.:238))

In these neighbouring Cape Town areas, Kaaps stabilised linguistically and took on the character for which it is recognised nowadays. The empirical finding by Kotzé (1984:42) that in BoKaap Afrikaans (or 'Afrikaans in the Malay Quarter', as he calls it) the typical linguistic characteristics of Kaaps are quantitatively embodied most strongly, is implicitly a confirmation of the status of the Bo-Kaap as the probable cradle of Kaaps. From the Bo-Kaap and District Six, Kaaps has, especially since the 1960s because of the Group Areas Act, but also long before that, expanded to different areas on the Cape Flats. The names 'BoKaap Afrikaans' ('Bo-Kaapse Afrikaans') and 'Cape Flats Afrikaans'? which came into use for Kaaps, thus prove to be precise indicators of the geographical demarcation and distribution of Kaaps.

\section{Typifying Kaaps in terms of its speakers}

In terms of its speakers, Kaaps is essentially sociolectic, rather than ethnolectic in nature. Accordingly, primarily the working class of Cape Town, including Cape Muslims and/or coloured people, but also white people, has always spoken Kaaps. To regard Kaaps as a marker of Coloured identity has therefore no solid base. These users of Kaaps include people native to Cape Town, but also persons from geographic areas outside Cape Town who, as residents of Cape Town, have over time acquired the ability to speak it.

Kaaps is, as indicated, chiefly a working class code. To put it differently: the cocoon of Kaaps is chiefly a working class cocoon. In the Cape Town city

7 Two spelling forms for the Afrikaans equivalent for 'Cape Flats Afrikaans' have emerged, namely 'Kaapse Vlakte-Afrikaans' (Karaan 2009) and 'Kaapsevlakteafrikaans' (Müller and Pistor 2011:36). 
centre, the suburbs of Cape Town and the Cape Flats where people from the working class or people from the middle class with a working class background live together, work together and on occasion socialize, there is a good chance that Kaaps or certain linguistic aspects of Kaaps will be used or heard. In terms of its dominant speaker corps, Kaaps could however be termed a colour variety of Afrikaans, i.e. a variety of Afrikaans which is traditionally associated with people of colour. As such, Kaaps is distinguished from other colour varieties of Afrikaans, such as the following:

a) the different subvarieties of the geolect Orange River Afrikaans which include Griqua Afrikaans and the colloquial Afrikaans of Namaqualand, Bushmanland, Richtersveld, Rehoboth and Riemvasmaak;

b) Flytaal or Tsotsi Afrikaans, a register variety which has Afrikaans as basis dialect and which is historically associated with young blacks in the residential areas of Johannesburg and

c) Black Afrikaans, an acquired colloquial variety which is geographically dispersed and which in its form shows traces of influence from one or other black language (Hendricks 2011:111).

To summarise, from a variationallinguistic perspective Kaaps is regarded as a variety of the dialect bundle Southwestern Afrikaans, which, as a form of colloquial Afrikaans, dates back to the seventeenth century influence of slaves on the formation of Afrikaans, which currently manifests mainly as a sociolect associated with the working class of the Cape Peninsula and which, in terms of its dominant speaker corps, can be typified as a colour variety of Afrikaans.

\section{THE LINGUISTIC NATURE OF KAAPS}

Contemporary Kaaps is characterised by particular phonological, lexical and grammatical phenomena (markers) and expressions. This intrinsic linguistic character reflects (a) the formative impact of, inter alia, English, Arabic, Malay and Indonesian on the DutchAfrikaans historical basis of Kaaps as well as (b) the linguistic influence of Standard Afrikaans and other forms of colloquial Afrikaans.

These aspects are consequently discussed and illustrated by means of examples from published texts in which Kaaps, or linguistic markers thereof, are incorporated, and sporadically also with reference to data recorded by myself. These sources are specified in full later in this paper under the heading "Database". ${ }^{8}$ My outline of the linguistic system of Kaaps is in essence a continuation of the linguistic description of Kaaps by myself (Hendricks 1978, 1996, 2012b) as well as the linguistic exploration of Kaaps by, inter alia, Klopper (1983), Kotzé (1984), Pheiffer

8. The examples used to illustrate the linguistic aspects of Kaaps in this section and further on, are presented as they appear in the sources they were extracted from. After each illustrative example, the source is indicated in accordance with the reference formula as specified in the database. For example: the source referencing for an illustrative example obtained from Adam Small's drama Kanna hy kô hystoe, is presented in the following way: daai < daardie (Small, KHKH, p. 85); for an item cited from Leonard Koza's short prose text 'Abdulla en Miena se stall'tjie' the referencing is as follows: wan' < want (Koza, 'Stall'tjie', p. 79). 
(1996), Van Rensburg (1989) and Ponelis (1989b, 1998).

Although the use of Kaaps is characterized by an alternation between variants typical of Kaaps and standardised variants, the description comprises a focus on the distinctive linguistic aspects of Kaaps. The developmental history of Kaaps is also taken into account and accordingly I deem it necessary to distinguish between older and more contemporary variants sporadically.

\section{The impact of English}

One of the most striking characteristics of Kaaps is the formative influence of English on its Dutch-Afrikaans base. The prominent nuances hereof are elucidated under (a), (b) and (c).

\section{(a) Insertion of direct borrowings from English}

Ek mean, sy was al seventy-eight, en nog altyd independant. Self town toe geloep, self haa' pension ge-draw ... Jus' shows you, you never can tell ... die dood kom ienagge tyd. (Otto-Sallies, Diekie, p. 49)

Hulle weet djy sal nie die local cops trust met 'n statement nie, toe stuur hy ouens uit sy jurisdiction om jou te test. (Son, 'Oom Sonnie', 10/4/2015)

Loop, en moenie iets try nie, want dan finish ek self die job. (Son, 'Oom Sonnie', 17/4/2015)

\section{(b) Morphological embedding of direct borrowings from English} racistgeid (Snyders, Political Joke, p. 55); unsettledgeid (Snyders, Political Joke, p. 56); ge-enter (Snyders, Die Burger Landelik, 10/5/2001); geleave (Small, KK, p. 81); gesurround (Snyders, Die Burger Landelik, 10/5/2001); hawkerkinners (Small, KHKH, p. 64); traffic cop-tjommie (Son, 'Oom Sonnie', 8/4/2015) (c) Prosodic Afrikaansification of inserted or morphologically embedded borrowings from English

erre-plein < aeroplane (Snyders, Die Burger Landelik, 24/5/2001); feks < facts (Otto-Sallies, Diekie, p. 64); gesettisfaid $<$ satisfied (Petersen, As die Son ondergaan, p. 25); koelek < collect (Snyders, Die Burger Landelik, 12/4/2001); draks $<$ drugs (Son, 'Oom Sonnie', 19/4/2015); pous-offies < post office (Petersen, 'In die hart van die stad'); sêd < sad (OttoSallies, Diekie, p. 48); sêdste (Small, KK, p. 80); vên < van (Son, 'Oom Sonnie', 9/4/2015)

Regarding this spontaneous mixing of Afrikaans and English, Kaaps represents, according to Ponelis (2009:12 ), the very high rate of English influence as prominent structural characteristic of demotic Afrikaans. This impact of English is reflected, as indicated later, in specific phonological, lexical and grammatical phenomena of Kaaps.

\section{Pronunciation phenomena in Kaaps}

Phonologically, Kaaps is strongly marked. Besides colloquial language markers such as procope, syncope and apocope as forms of speech sound omission (see d), epenthesis and paragoge as forms of speech sound addition (see e), unrounding (see f) and enclisis (see g) which Kaaps has in common with other forms of colloquial Afrikaans, the phenomena at (h) collectively can be called distinctive pronunciation markers of Kaaps:

\section{(d) Omission of speech sounds}

- Syncope:

atappels < aartappels (Koza, Stall'tjie, p. 79); ammal < almal 
(Small, KHKH, p. 21); sukke < sulke (Small, KHKH, p. 44); t'rug < terug < terug (Son, 'Oom Sonnie', $19 / 4 / 2015)$

- Apocope:

$k \hat{o}<\operatorname{kom}$ (Small, KHKH, p. 63); soelang < solank (Otto-Sallies, Diekie, p. 66)

\section{(e) Addition of speech sounds}

- Epenthesis:

- Nasal epenthesis:

klamvier < klavier (Small, $K K H$, p. 61), pampiere < papiere (Small, KHKH, p. 65)

- Schwa-epenthesis (which leads to syllable addition):

oepeslaan < oopslaan (Small, KHKH, p. 32)

- Paragoge in Afrikaans as well as English words:

lakent < laken (Small, KHKH, p. 55); oefent < oefen (Son, 'Oom Sonnie', 17/2/2013); smaail $t<$ smile (Small, $K K H$, p. 62), spoil $t<$ spoil (Small, $K K H$, p. 62)

\section{(f) Unrounding}

hiening < heuning (Koza, Stall'tjie, p. 79); piere < pure (Otto-Sallies, Diekie, p. 28); ytgegooi < uitgegooi (Son, 'Oom Sonnie', 19/4/2015)

\section{(g) Enclisis plus omission and/or amendment of speech sounds}

- Enclisis of die:

innie < in die (Koza, Stall'tjie, p. 82 ); ommie < om die (Snyders, Beeld PLUS, 17/2/1998, p. 4)

- enclisis of dit:

assit < as dit (Snyders, Beeld PLUS, 17/2/1998, p. 4); vertellit < vertel dit (Snyders, Beeld PLUS, 17/2/1998, p. 4)
- enclisis of het:

hie' $t<$ hier het (Snyders, Beeld PLUS, 17/2/1998, p. 4)

- enclisis of is:

daais < daai is (Koza, 'Stall'tjie', p. 84; Otto-Sallies, Diekie, p. 60); djy's $<$ djy is (Otto-Sallies, Diekie, p. 60)

- enclisis of nie:

issie < is nie (Snyders, Beeld PLUS, 17/2/1998, p. 4); mossie < mos nie (Otto-Sallies, Diekie, p. 59); waggie $<$ wag nie (Koza, 'Stall'tjie', p. 79)

(h) Distinctive pronunciation markers of Kaaps

- /e/-raising:

briek < breek (Small, KMK, p. 59); kniehoegte < kniehoogte (Snyders, Die Burger Landelik, 29/3/2001); uitgevriete < uitgevrete (Son, 'Oom Sonnie', 9/4/2015); wiet < weet (Son, 'Oom Sonnie', 19/4/2015)

- /o/-raising: loep < loop (Otto-Sallies, Diekie, p. 65); oek < ook (Small, KMK, p. $64) ;$ soe < so (Son, 'Oom Sonnie', 19/4/2015)

- Schwa lowering $(/ \mathrm{\partial} />/ \mathrm{a} /)$, especially with $g e$ - and $-i g$ :

biesag < besig (Otto-Sallies, Diekie, p. 21); biesagheid < besigheid (Koza, 'Stall'tjie', p. 81); galoef < geloof (Small, KMK, p. 36); galukk $a \mathrm{~g}$ $<$ gelukkig (Small, KMK, p. 57); haastag < haastig (Koza, 'Stall'tjie', p. 79); last $a \mathrm{~g}<$ lastig (Otto-Sallies, Diekie, p. 26)

- /a:/-shortening (/a:/ > /a/): an $<$ aan (Otto-Sallies, Diekie, p. 61); angan < aangaan (Otto-Sallies, Diekie, p. 70); gan < gaan (Small, $K K H$, p. 59); gegat < gegaan (OttoSallies, Diekie, p. 21); Kap < Kaap (Small, KKH, p. 59) 
- Abolition of ablaut with formation of plural:

Biesagheite i.p.v. besighede (Small, $K H K H$, p. 67); skippe i.p.v. skepe (Small, DS)

- Post-vowel /r/-omission:

Hoo' hie', ons miet hie' oppie hoek.

(Otto-Sallies, Diekie, p. 66)

Dja ek sê vi' djou lat ons $m a$ ' hou ... (Small, KMK, p. 64)

- /j/-affricatisation:

djammer < jammer (Son, 'Oom Sonnie', 10/4/2015); djol < jol (Son, 'Oom Sonnie', 10/4/2015); djuis < juis (Son, 'Oom Sonnie', 19/4/2015); djulle < julle (Son, 'Oom Sonnie', 8/6/2011); djy moet djou palys-hys (Small, $K M K$, p. 25)

- Palatalization in the diminutive ending:

bietjie [bit $\left.\int \mathrm{i}\right]$ i.p.v. [bici]; karrentjie [karənt $\left.\int i\right]$ i.p.v. [karici]; stertjie [ste:t $\left.\int i\right]$ i.p.v. [sterci]

- Homorganic end cluster simplification in Afrikaans as well as English lexical items:

- in Afrikaans lexical items:

/lt/ à /l/: gel' < geld (Otto-Sallies, Diekie, p. 66)

/nt/ à /n/: mon' < mond (OttoSallies, Diekie, p. 34); wan' < want (Koza, Stall'tjie, p. 79)

/mp/ à /m/: klom' < klomp (OttoSallies, Diekie, p. 62)

- in English lexical items:

/ld/ à /l/: ol' < old (Small, KMK, p. 89); tol' < told (Small, KMK, p. 53)

$/ \mathrm{nd} / \rightarrow / \mathrm{n} /:$ spen ${ }^{\prime}<$ spend $($ OttoSallies, Diekie, p. 35)
- Rhotacism:

- Rhotacism of an intervocalic /d/:

byrie [bəiri] < by die (OttoSallies, Diekie, p. 62); Joremeisies $<$ Jodemeisies (Small, KKH, p. 62); mirrag < middag (Koza, Stall'tjie, p. 80); mirrel < middel (Trantraal, Rapport Weekliks, 10 January 2016, p. 15)

- Rhotacism of an intervocalic /d/, following hypercorrect voicing:

skuite $\rightarrow$ [skəidə] $\rightarrow \quad$ [skəirə], soldate $\rightarrow$ [solda:də] $\rightarrow$ [solda:rə] (Kotzé 1984)

- Rhotacism plus nasal apocope:

gaan dit $\rightarrow \quad$ [garət], gekom het $\rightarrow$ [xəkərət] (Kotzé 1984)

- Vowel lengthening plus post-vowel /r/-omission:

sôg < sorg (Trantraal, Rapport Weekliks, 10 January 2016, p. 15); wôd < word (Trantraal, Rapport Weekliks, 10 January 2016, p. 15)

\section{The use of lexical items and expressions in Kaaps}

Kaaps is not only strongly marked regarding pronunciation, but especially also lexically. Besides the frequent use of English lexical items, the intertwining of lexical items which are linked to Islam and which are etymologically of Arabic, Malay and Indonesian origin, is also typical of Kaaps. Many of these items, including those supplied in (i), were included in the tenth edition of the Afrikaanse Woordelys en Spelreëls (Taalkommissie 2009). ${ }^{9}$ The examples at $(\mathbf{j})$ illustrate the literary reflection of these items.

9 Lexical items linked to Islam (i.e. Muslim Afrikaans lexical items) which were included in the 2009 edition of the Afrikaanse Woordelys en Spelreëls, are listed in Kotzé (2010:170-171) and Kotzé (2012:43). 


\section{(i) Lexical items from the AWS}

\section{linked to Islam}

asser (prayer time), boeja (father), boeka (break the fast), djoemoea (mosque service), do'a maak (intercede), gadj (pilgrimage to Mecca), garaam (prohibited), kanalla (please), koefia (fez), Koer'aan (Qu'ran), madrassa/madressa (religious school), magriep (prayer time), nasara (a Christian), oemi (mother), salaah maak (pray), sjoekran (thank you), trammakassie (thank you)

\section{(j) The literary reflection of lexical items linked to Islam $^{10}$}

aleikoem salaam $\{$ May there be peace and blessings on you $\}$ :

Aleikoem salaam. Ja, Oemie, ek sal vi'

Mamma sê. (Otto-Sallies, Diekie, p. 25)

Bismallah \{In the name of Allah\}:

'Bismallah,' sê Pang en val weg. (Koza, 'Stall'tjie', p. 85)

gamdoeliela < algamdoeliela $\{\mathrm{By}$ the grace of Allah (we are well)\}:

Gamdoeliela, my kin'. (Otto-Sallies, Diekie, p. 14)

iensha'alah \{if Allah so wills\}:

Iensha'allah, Oemie. Oemie, is Koelie hiesa? (Otto-Sallies, Diekie, p. 25)

kanalla \{please\}:

"n Koppie tee, kanalla bokkie,' sê Pang en hy plak homself op 'n kombuisstoel neer. (Koza, 'Stall'tjie', p. 83)
The lexical terms supplied in $(\mathbf{k}),(\mathbf{l}),(\mathbf{m})$ and $(\mathbf{n})$ are also typical of contemporary Kaaps. The lexical items in (k) are commonly in use among Kaaps speakers, while those in (1) more specifically relate to the Cape underworld (gang activies, prison life, and alcohol and drug abuse). The items in (m) Kaaps has in common with Flaaitaal/Tsotsi language. The items in (n) are phonetic modifications or contractions of words and/or expressions, which, because of a high frequency of usage, with time have achieved lexical (word) status. Lexical items such as those in (o) were earlier highly customary among speakers of Southwestern Afrikaans in general and among Kaaps speakers in particular, but its usage has decreased over the past three decades, probably due to the impact of Standard Afrikaans. Some of these items in (o) have found their way as lemmas in the Woordeboek van die Afrikaanse Taal (WAT) (Hendricks 2012b:104).

The items in (p) are representative of typical sayings/expressions among speakers of Kaaps.

\section{(k) Commonly used lexical items}

afbene/afbiene (adj.) \{broke\}:

Om afbene te wees, is om platsak te wees. (Rapport, 5/11/2012)

afsak (v.) $\{$ say $\}$ :

Ek haat houtvloere, dink ek, en $s a k$ af: 'Die kind is al weer honger.' (Son, 'Oom Sonnie', 20/4/2015)

Hy (...) sak af: Moenie vir hulle kop toe vat nie. (Snyders, Political Joke, p. 16)

\footnotetext{
10 In the elucidation of lexical items and expressions supplied here and further also at (k-p), three kinds of brackets are used, i.e. square brackets: [ ], round brackets: ( ) and face brackets: \{ \}. Pronunciation is indicated in square brackets, part of speech status and/or usage in round brackets, and meaning in face brackets. Pronunciation is only supplied in cases where the pronunciation of lexical items might not be clear from the orthography. Examples: bad [bat] (v.) \{squander\}; lai-lai/laai-laai (adj.) \{bedeviled; unreasonable\}.
} 
as an/as-an (adj.) \{not bothered\}:

My pa is al klaa' soe as an. (Otto-

Sallies, Diekie, p. 52)

Toe ons byrie dokter kom, was ek bietjie verbaas dat hy so as-aan is. (Snyders, Nursie)

aweh! (general customary form of greeting):

'Aweh, my antie en my uncle,' sê die laaitie. (Son, 'Oom Sonnie', 8/6/2011)

'Aweh, Tommy, vir wat hang jou bek dan so?' wil Gammie weet. (Son, 'Oom Sonnie', 19/8/2012)

$\boldsymbol{b a d}$ [bat] (v.) \{squander\}:

My geld het gebad. (Own recording)

bad [bat] (v.) \{be imprisoned; serve prison sentence $\}$ :

'Hy moet vir drie maand gaan bad.'

(Own recording)

berk [bə:g] (n.) \{boyfriend\}:

Hy kon nou net nie die hardegat kallit met die boep weerstaan nie, al wou sy dan nou haar berk laat doodmaak. (Son, 'Oom Sonnie', 20/4/2015)

djep $[\mathrm{d} z \varepsilon p](\mathrm{v}).\{$ steal $\}$ :

My ou-hulle gat na 'n eat 'n treat toe, ma' hy gattie onse kar vattie. (...) Soe ek gattie cab djep, dan kan ons 'n bietjie skud. (Otto-Sallies, Diekie, p. 65)

duidelik/dydelik (adj.) \{undeniably good; to be excited about $\}$ :

$\mathrm{Ja}$, jong, oom Sonnie is weer aan die brand! Duidelik. Boeta Sonnie is duidelik. (Son, 'Ditjies en Datjies', 23/4/2010)

Ek is ou B, maar almal ken my as Blackjack. Dis 'n duidelike naam vir my. (Son, 8/12/2008)

eish [əi $\left.\int\right] /\left[\varepsilon: \int\right]$ (interjection from isiZulu) \{oh my word; wow $\}$ :
'Eish, ek het my foon in die kamer vergeet en kan die gedoente dus nou nie time nie,' skel ek myself uit. (Son, 'Oom Sonnie', 21/4/2015)

'Eish, dis seker nou sy ma en suster, want ek het kliphard geskreeu ...' (Son, 'Oom Sonnie', 22/4/2015)

entjie(s) [ent $\left.\int \mathrm{i}(\mathrm{s})\right] \quad / \quad\left[\varepsilon: \mathrm{nt} \int \mathrm{i}(\mathrm{s})\right] \quad$ (n.) $\{$ cigarette(s)\}:

Giemba, het djy nie 'n entjie daa'nie, ek smaak 'n skyf, man. (Otto-Sallies, Diekie, p. 11)

En hie' tjek ek 'n bottle Vat 69 en 'n gros entjies sal djou van plan laat verander. (Son, 'Oom Sonnie', $13 / 11 / 2012)$

gaga [gaga] (adj.) \{excited\}:

Dit is heel normaal vir couples om nie gaga te gaan oor dieselfde goed nie. (Son, 'Antie Mona', 21/2/2014)

gevaa $(\mathbf{r}) l i k$ (adv. of degree) \{very\}:

Daai kin' was mos gevaa'lik lief vi' Oemie. (Otto-Sallies, Diekie, p. 49)

ghibbes [gabas] (n.) \{boy-friend/ girl-friend $\}$ :

Daai tyd was sy mos my ghibbes. (Own recording)

habba $\{$ nothing/no\}:

Ek het lus vir roek ma' het habba gel' vi' entjies. (Own recording)

hollang/holhang (v.) \{hang around; relax; lay about $\}$ :

Ons kannie wêk kry nie, nou hollang ons ma' op die winkelstoep. (Own recording)

karra/kyra or ky'ra-da (interjection) $\{$ look there $\}$ :

karra (Petersen, 'Op Nuweland')

En ky'ra-da, hie bring dié ekke 'n oulappie hys toe. (De Vries, Baie melk, p. 96)

kwaai: (adj.) \{good; nice; fantastic\}: 
Oom Sonnie, jou storie oor die meisies is kwaai. (Son, 'Ditjies en Datjies', 7/9/2009)

Ek like jou style. Kwaai suit, kwaai bril ... jy verdien seker baie? (Son, 'Oom Sonnie', 11/3/2010)

lai-lai/laai-laai (adj.) \{bedeviled; unreasonable\}:

Nee, (...) sy's net lai-lai, skoons beneuk (De Vries, Baie melk, p. 76)

lam/laam/lam it yt (v.) \{sit; hang around $\}$ : Ek gat lieweste' 'n bietjie by Mileshulle lam (Otto-Sallies, Diekie, p. 28)

Ek sit met 'n bottel witwyn, net vi my. Hy lam it yt met 'n halwe bottel whiskey (Trantraal, Rapport Weekliks, 10 January 2016, p. 15)

meneer (form of address or reference for a teacher; pl.: menere):

Anthony se jonger suster en sy enkelma is tussen die laaste groep wat by die twee onnies loop. 'Bye, meneer,' sê die jong Mekkie. 'Dankie vir alles wat meneer-hulle vir ons, vir Anthony, gedoen het.' (Son, 'Oom Sonnie', 9/4/2015)

mobile [mœuba:il] (n.) \{small shop on wheels\}:

Hoe kan die selfoon-netwerke R5 vra vir lugtyd, maar dan kom jy by die mobile wat R6 of selfs meer vra? (Son, 'Ditjies en Datjies', 28/4/2010)

moet instead of met as preposition:

Nei, (...), ons ammal het al moet hom gepraat. (Otto-Sallies, Diekie, p. 57)

Ou Rocks, ek sal laaiks om saam moet jou te gat ... (Otto-Sallies, Diekie, p. 62)

$\operatorname{mol}[\mathrm{mo:l}]$ (v.) \{in a hurry to get away; push forward competitively\}:

'O bliksem!' gil Grompot. Hy en Baksteen gooi hul gewere neer en mol vir hul bakkie. (Son, 'Oom Sonnie', 29/5/2015)
Cool Daddy en sy vier lyfwagte spring gelyk op en mol vir die venster wat op die shebeen afkyk. (Son, 'Oom Sonnie', 5/10/2012)

oorlams (adj.) \{difficult; bad-tempered $\}$ : En nou is my pa oek soe oo'lams byrie hys. Hy wil vi' hom soe difficult hou. (Otto-Sallies, Diekie, p. 51)

nwaatas praat talk nonsense\}:

Moetie nwaatas praat'ie, man. (OttoSallies, Diekie, p. 74)

pel/pêl/pellie (item used to address, or to refer to, a friend/mate):

Djy's sieke' bang Boeta Dieks willie mee' jou pel wiese (Otto-Sallies, Diekie, p. 60)

Djy's 'n lucky ou, djy biete' vashou an Ice, of anneste', pellie, is sy myne. (Otto-Sallies, Diekie, p. 56)

Toe sy twee pêlle later kom blyplek soek het, het hy besluit om hulle tydelik hier te akkommodeer ... (Son, 'Oom Sonnie', 24/6/2012)

papgeld (n.) \{payment of child support Djy moet tog uitvind wat van daai vrou geword het by wie Abdullah die kind het. Ek wonne' of hy haa' papgeld betaal. (Koza, 'Stall'tjie', p. 80)

sterkgevreet/sterkgevriet wees (adj.) $\{$ (presumably) ready to fight/attack or ready to defend $\}$ :

Die personeel by sekere hospitale is sterkgevriet met die pasiënte, en dan word jy goed sleggesê. En as jy eerste sterkgevriet is, weier hulle om jou te help. (Son, 7/8/2013)

Miena het nooit vi' my tale gegie nie. Va'dag is sy soe stê'k gavriet, êrens moet daa' iets ve'kee'd wies. (Koza, 'Stall'tjie', p. 84) 
sterkgevriet (vocative for someone (presumably) ready to fight/attack or ready to defend):

'Wie se nekke (gaan jy omdraai)? Doennit sommer nou, jou sterkgevriet,' sê 'n diep stem hier vlak agter die onnies. (Son, 'Oom Sonnie', 9/4/2015)

tanie (n.) $\{\mathrm{mom}\}$ :

Moetie mal wiesie, daai tanie (van Ice) dink Ice is nog 'n baby. (OttoSallies, Diekie, p. 43)

Vanoggend (...) was dit net my tanie wat my omhels en gesoen het. (Son, 'Oom Sonnie', 24/6/2012)

Is my taanie wat haar weer bad luck hou, man. (Son, 'Oom Sonnie', $19 / 8 / 2012$ )

tietie/tie' (item used to address, or to refer to, an older sister respectfully):

Tie Lyla soek somme' wee' trouble. (Otto-Sallies, Diekie, p. 64)

As Diekie of Ice met die grootmense praat, (...), moes hulle praat van Tie Sieda, Tie Koelie - 'n vorm van respek (Otto-Sallies, Diekie, p. 17)

Phone vi' jou tietie en vra vi' haa' om twiehonne't samosas te bak, kanalla. (Otto-Sallies, Diekie, p. 49)

toppie (n.) $\{\mathrm{dad}\}$ :

Gaan jou toppie vir djou geld voorskiet? (Son, 'Oom Sonnie', 24/6/2012)

Sy stief-toppie bly moan omdat hy nie kan werk kry nie. Sy ma is maar die een wat al die jare die toppie moes paai en vra om geduldig te wees. (Son, 'Oom Sonnie', 2/11/2011)

twaisfai [twaisfa:i] (v.) \{argue; fall out with\}:

Kyk, met sy 'rules is rules' was ek ga lus om te twaisfai nie, want wat baat 'n getwis tog met 'n onversetlike iemand? (Rapport, 23/3/2014)

Nie eens Stonehill (een van die 'beterder' buurte in Ravensmead) se sturvy span met hul pinkie-innielug-geite kon dáármee ge-twaisfai het nie. (De Vries, Baie melk, p. 28)

versin (adj.) \{crazy; robbed of your senses\}:

Djy's soe geroek, djy's skoon ve'sin. (Otto-Sallies, Diekie, p. 60)

Kyk hoe ve'sin lyk djy al. Djy act al mal van allie drugs. (Otto-Sallies, Diekie, p. 60)

$\operatorname{vertel}(\mathrm{v}) .\{$ insult; tell off $\}$ :

Ek sê, Rocky, djy ka' mossie vi' my soe ve'tellie, my broe' ((Otto-Sallies, Diekie, p. 59)

Koelie, ...., ek het gedink ek dien iets goet, nou kom djy my soe ve'tel. (Otto-Sallies, Diekie, p. 64)

volmaak (v., with the emphasis on the first syllable) $\{$ say/tell; inform; convince $\}$ :

Ek sal hulle volmaak dat tussen dié kant vannie mens en daai kant vannie geld issaar liëwe; tussenin issaar net onkunde. (Snyers, Die Burger Landelik, 6/6/2001)

'As dié dinge van jou nie ent kry nie, sal ons moet praat oor jou toekoms,' het Small se baas hom volgemaak. (Snyders, Onopgeloste karma)

witbene/witbiene (adj.) \{dead, deceased $\}$ : Twee onskuldige mense is dood as gevolg van haar verraad. Twee mens is witbene omdat sy met mense se gevoelens speel. (Son, 'Oom Sonnie', 21/2/2012)

Djy's 'n sleg ding wat agter vroumense skuil om jou vuilwerk te doen. But ek promise jou: vanaand is djy witbene. (Son, 'Oom Sonnie', 1/3/2012)

Anyway, ou Jamesie is lankal witbene. 
(Son, 'Oom Sonnie', 13/11/2012)

woelag (adj.) \{impressive; superb\}:

Dis aand en die jol is woelag. (Son, 'Oom Sonnie', 25/2/2011)

Daai goose het mos 'n pa' woelage biene. (Own recording)

ytstiek/uitsteek/uitstiek (v.) \{arrive, turn up\}:

Die cops het eers ure later uitgesteek toe Samson weens die nokke teen sy kop gesterf het. (Son, 3/3/2014)

Ammal stiek hie' yt eight o' clock sharp. (Otto-Sallies, Diekie, p. 66)

Oral waa' djy uitstiek, is daar bloed en derms. (Son, 'Oom Sonnie', $12 / 11 / 2012$ )

(l) Kaaps words linked to prison life, gangsterism, and alcohol and drug abuse

afhaal (v.) \{kill; get rid of $\}$ :

Djy, sal dit seker ook funny vind dat ek gehuur is om Melody af te haal, nè? (Son, 'Oom Sonnie', 20/1 1/2012)

'Nee,' sê die hit man. 'Die Man het gesê ons moet jou kom afhaal om te wys niemand mors met hom nie.' (Son, 'Oom Sonnie', 10/6/2011)

boere (n.) \{police officers\}:

Moenie worry nie, alles is klaar georganise. Os is reg virrie boere. (Son, 'Oom Sonnie', 16/4/2015)

Nou weet djy hoekom Claremont se boere netnou by die skool was. (Son, 'Oom Sonnie', 16/4/2015)

dikding (n.) \{leader/bigwig $\}$ :

Môre stuur die dikdinge net nog jongens om hul vuilwerk te doen. (Son, 'Oom Sonnie', 17/4/2015)

Die gangster-dikdinge het mos geld, flashy karre, groot huise, en spoil die mense met mahala kos, drank en rent-geld. (Son, 'Oom Sonnie', 9/4/2015)

dik gesuip (adj.) \{dead drunk\}:

Hy's dik gesuip en het g'n vervoer om by die huis uit te kom nie. (Son, 'Oom Sonnie', 12/11/2012)

'Dik gesuip, maar ken steeds djou storie. Soe ken ek djou mos, ...' (Son, 'Oom Sonnie', 12/11/2012)

gangster(s) [gæystə(s)] (n.) \{gangster(s); mobster(s)\}:

Die gangster knik sy kop en Keon sê verder: 'Djy bieter nie vir my lieg nie (...)’ (Son, 'Oom Sonnie', 21/6/2010)

Die gangsters skiet oral en die arme mense sterf soos vlieë (Son, 'Oom Sonnie', 16/4/2015)

ganja [gandza:] (n.) \{dagga; marijuana\}: Hoekom kan die cops net nie die Rastas uitlos nie? Hulle raid ons aanmekaar vir ganja wat ons self nie gemaak het nie. (Son, "Ditjies en Datjies", 18/9/2009)

ganja-poppie(s) [gandza:popi(s)]

\{woman/women who smoke dagga\}:

Hou op, julle ganja-poppies. (Son, 'Antie Mona', 6/4/2009)

$\boldsymbol{g a t t a}(\boldsymbol{s})[\mathrm{xata}(\mathrm{s})]$ (n.) \{police officer(s)\}:

'Ek sê, relax, my broe', is ma' nettie gattas wat hulle stêk wil hou.' (OttoSallies, Diekie, p. 74)

'Die gattas gaan weet waar om te soek.' (Son, 'Oom Sonnie', 20/11/2012)

ghaazie/gasielam: \{best friend\}

Ek mean, my ghaazie, is al twie maan'e manieng-al, ma' ek kan noggie reg slaap'ie. (Otto-Sallies, Diekie, p. 86)

mang [man] (n.) \{prison\}: Jenna is in die mang en die Ding 
is oorsee. (Son, 'Oom Sonnie', 20/4/2015)

mang [may] (v.) \{serve prison sentence; detained in prison\}:

Dis net ' $n$ jammerte Bones en Koppe gaan vir ' $n$ jaar of wat moet mang. Daar sal egter goed agter hulle gekyk word in die tronk. (Son, 'Oom Sonnie', 7/4/2015)

mapoesa(s)/mapoeza(s) $\quad$ (n.) $\quad$ \{police officer(s)\}:

Daai's 'n mapoesa daai. (Snyders, Political Joke, p. 37)

'Wat soek jy met die mapoezas hier?' vra hy aan Don. 'Jy weet mos hoe het hulle my in die apartheidsjare gejag.' (Son, 'Oom Sonnie', 10/2/2010)

$\operatorname{mert}(s) \quad[\operatorname{mə:t}(\mathrm{s})] \quad(<\operatorname{merchant}(\mathrm{s}))$ $\{$ drug merchant(s) $\}$ :

Logan sit gemaklik in 'n stoel, terwyl die mert by die breë venster uitstaar. (Son, 'Oom Sonnie', 28/1/2013)

Logan weet nie hoekom die merts die goed genommer het nie. (Son, 'Oom Sonnie', 28/1/2013)

nommers (n.) \{prison gangs\}:

Ek latie die nommers my intrek nie (...) Daa's sommige vannie beamptes wat die nommers se werk doen. (Son, 'Oom Sonnie', 1/3/2015)

Hierdie bewaarders meen hulle is nommers, maar in ons oë is hulle franse, nie-bendes. (Son, 'Oom Sonnie', 10/5/2010)

piemp (v.) \{betray; split on $\}$ :

'Hulle is bang hulle stel hulle laaities se lewe in gevaar as hulle op die merts piemp,' voeg hy by. (Son, $16 / 2 / 2012$ )

piemper (n.) \{traitor; blabber $\}$ :

Die piemper het sy lewe gewaag om die inligting uit te kry ... (Son, 'Oom Sonnie', 16/4/2015) sabela [sabela] (v.) \{speak prison lingo $\}$ :

Niemand wil met my oor die nommer 28 sabela (praat) nie. Hulle is bang hulle ken nie die reëls en wette van die nommer nie. (Son, 'Oom Sonnie', 10/5/2010)

Soos jy die klip kap, word daar oor die nommer sabela (gepraat). Ons knap ons reëls en wette van die nommer op. (Son, 'Oom Sonnie', $7 / 7 / 2010)$

satmaak (v.) $\{$ kill $\}$ :

Djy moet vi'staan dat djy op die groot papa se tone getrap het die dag toe djy besluit het om Mongesh sat te maak. (Son, 'Oom Sonnie', $13 / 11 / 2012$ )

smokkie(s) (n.) $\{$ shebeen(s)\}:

Dodelike geveg in smokkie lei tot die dood ... (Son, 'Oom Sonnie', $6 / 6 / 2011)$

Daar's nie onnodige gun-skietery nie. Niks gangsters op die straathoeke of lawaaierige smokkies by elke tweede huis nie. (Son, 'Oom Sonnie', 28/1/2013)

tanne-nat (adj.) \{drunk; tipsy\}:

Ammal was lekka tanne-nat by John se rampaa'tie. (Own recording)

$\boldsymbol{t i k}(\mathrm{n}$.$) the drug crystal methamphetamine \}$ : Hier (...) is baie jong mense wat drank en tik gebruik. Jong meisies word vir tik misbruik. (Son, 'Antie Mona', 7/5/2008)

$\boldsymbol{t i k}$ (v.) \{use the drug tik\}:

'n Ma van drie beweer haar man tik en het haar so amper-amper al aan die brand gesteek ... (Son, 21 Oktober 2011)

Die trug trade daar is hoog en die jonges en oues tik. (Son, 'Oom Sonnie', 28/1/2013) 
tikkop (n.) \{someone who uses the drug tik\}:

Ek en my kind se pa het voorgekom vir non support. (...) Hy is 'n tikkop en wil nie werk nie. (Son, 'Antie Mona', 21/1/2013)

tjoef (n.) \{the drug tik\}

Sy is 'n tikkop-prossie wat haar lyf vir tjoef verkoop. (Son, 6/6/2014)

tjoef (v.) \{use the drug tik\}:

Riaan, wat al tien jaar tjoef, sê hy dink nie hy het 'n dwelmprobleem nie. (Son, 18/2/2014)

tjoefer (n.) \{someone who uses the drug tik\}:

Maar volgens die tjoefer dwing geld, 'n slang en die diere op papiergeld hom om sy ouma so te verrinneweer. (Son, 18/2/2014)

tjoefkop (n.) \{someone who uses the drug tik\}

Die tjoefkop se girlfriend, Candice Julius, beaam Alexander se storie. (Son, 11/3/2011)

uithaal (v.) \{kill; get rid of $\}$ :

Djy inform my dat djy hier is om my uit te haal. (Son, 'Oom Sonnie', $13 / 11 / 2012$ )

En wie is die ander jongens wat oek suppose is om my uit te haal? (Son, 'Oom Sonnie', 13/11/2012)

vedala [fəda:la] (v.) \{kill, murder\}:

Toe hy sien, skree hy dadelik: 'Ou Pieter, jou broerse wil my vedala!' (Son, 'Oom Sonnie', 1/4/2010)

'Djy maak djou laat as djy dink djy ga my soe easily vedala, bru,' gil die dude. (Son, 'Oom Sonnie', 21/2/2012)

vedala [fəda:la] (adj.) \{not as desired $\}$ :

Ôs bly by my oupa en sy hys lyk vedala, no question. (Trantraal, Rapport Weekliks, 7 February 2016, p. 15)

uitpype/ytpype (adj.) \{unconscious\}

By streat fighting is die enigste doel net om jou opponent so gou moontlik bebloed en uitpype op die grond te kry. (Son, 'Oom Sonnie', 25/2/2011)

Die gangster is amper ytpype, maar Keon hou hom aan sy keel orent en praat in sy erg bebloede gesig. (Son, 'Oom Sonnie', 21/6/2010)

zol (n.) \{dagga cigarette $\}$ :

Dagga-aktivis rook 'n zol tydens regstreekse onderhoud. (Son, $12 / 5 / 2015$ )

Daar is 26 dagga-zolle in sy huis gekry. (Son, 3/3/2014)

(m) Kaaps words with equivalents in Flaaitaal/Tsotsi language

bra/bla/broe $(\boldsymbol{r}) / \boldsymbol{b r u}$ (item used to address, or to refer to, a friend/mate):

Hys'ie actually onse bra nie ... (OttoSallies, Diekie, p. 61)

'Ou Dieks,' sê hy, 'djy's 'n ghrên bla ...' (Otto-Sallies, Diekie, p. 61)

My broe', ek het vi' Oemie ge-promise ... (Otto-Sallies, Diekie, p. 61)

Ek hoor net: 'Stadig, stadig, my broer.' (Son, 'Oom Sonnie', 1/4/2010)

'Djy maak djou laat as djy dink djy ga my soe easily vedala, bru,' gil die dude. (Son, 'Oom Sonnie', 21/2/2012)

buddy (item used to address, or to refer to, a friend/mate):

'Laai jou buddy in jou kar,' sê hy.

(Son, 'Oom Sonnie', 10/6/2011)

gwarra (v.) \{mock; tease $\}$ :

Die laaities likes om meka' te gwarra.

(Own recording)

gwarra (n.) $\{$ mockery\}:

(Hulle) het niks van dié speelse 
gwarra gehou nie. (Snyders, Die Burger Landelik, 23/8/2001)

joint (n.) \{house $\}$ :

Ek mean, ek ko' by julle joint, ek kan mos sien hoe jou ma met jou ou sukkel oor sy roekery. (Otto-Sallies, Diekie, p. 61)

mahala (adj.) \{free of charge :

Die gangster-dikdinge het mos geld, flashy karre, groot huise, en spoil die mense met mahala kos, drank en rent-geld. (Son, 'Oom Sonnie', 9/4/2015)

$\operatorname{sharp}$ (adj.) \{It's good $\}$

'Kom maak vanaand 'n draai, sê John. 'Sharp, my bra!' antwoord Billy. (Own recording)

skanghagha/skanghaka/skankie(s) (adj.) \{everything is fine/in order\}:

'Nei, vir my klink die plan nogal skanghagha!' sê Punkie en vat nog 'n teug aan die daggapyp. (Son, 'Oom Sonnie', 24/6/2012)

Salute ouens, man. Okay, oek oek. Ek tel op julle is skanghaka, man. (Die Burger, 1/4/2002)

tjerrie(s) (< cherry) (item used to address, or to refer to, a girlfriend):

Ou Chris het seker die lelikste meisie denkbaar uit die bondel gevat en haar sy vaste tjerrie gemaak. (Son, 'Oom Sonnie', 12/8/2012)

$\mathrm{Ek}$ meen, daar is só baie lekker tjerries hier. (Son, 'Oom Sonnie', 12/8/2012)

tjommie (< chummy/chommie) (item used to address, or to refer to, a friend/ mate):

Ek sal 'n tjommie van my vra of hy nie vi' djulle plek hettie. (Koza, Stall'tjie, p. 82)

'Hy is bad news,' sê Gus se traffic cop-tjommie. (Son, 'Oom Sonnie', $8 / 4 / 2015)$
Antie (...) dan sê sy vir haar tjommies en dan kom soek hulle my op. (Son, 'Antie Mona', 8/12/2009)

\section{(n) Lexified phonetic modifications and/or contractions}

dai < daardie (Trantraal, Rapport Weekliks, 10 January 2016, p. 15)

daai < daardie (Small, KMK, p. 85; Son, 'Oom Sonnie', 20/4/2015)

daantoe $<$ daarnatoe (Otto-Sallies, Diekie, p. 66)

ganiemant/ganiemand < geen iemand (Small, KKH, p. 60)

gwaan < go on (Petersen, 'Op Nuweland'; Small, $K H K H$, p. 41; Small, $K M K$, p. 71)

halloep/ha'loep < hardloop (Koza, Stall'tjie, p. 84; Otto-Sallies, Diekie, p. 44)

hientoe $<$ hiernatoe (Otto-Sallies, Diekie, p. 62)

hiesa < hierso (Otto-Sallies, Diekie, p. 62)

$\boldsymbol{h} \hat{\boldsymbol{o}}<$ hoor (Petersen, 'Op Nuweland')

hoelaha < haai, o Alla (Own recording) of hoelie-ha (De Vries, Baie melk, p. 30)

ka-re $<$ kaarte (Petersen, 'Rotjies')

karra/kyra < kyk daar! (Petersen, 'Op Nuweland)

nemma < netnoumaar (Petersen, 'Bandeloos')

nemmatjies < netnoumaartjies (Petersen, As die son ondergaan, p. 24; Otto-Sallies, Diekie, p. 53)

ôs/os < ons (Trantraal, Rapport Weekliks, 7 February 2016, p. 15)

slytel < sleutel (Small, KKH, p. 60)

soema < sommer (Small, KKH, p. 62; Son, 'Oom Sonnie', 20/4/2015) 
waantoe < waarnatoe (Snyders, Political Joke, p. 2)

whit [wo] < word [vort] (Otto-Sallies, Diekie, p. 64)

whit $[\mathrm{wət}] /[\mathrm{wə:t}]<$ woord [vort] (Kotzé $1984)^{11}$

\section{(o) Lexical items from earlier Kaaps:}

- Substantives:

- Countable nouns: dieners ( $<$ dienders) for police officers (Small, KMK, p. 86); dogte instead of dokter (Small, KHKH, p. 63); krant instead of koerant (Small, KMK, p. 85)

- Uncountable (mass) nouns knoflok instead of knoffel (Own recording)

- Nominals ending on -entheid: boekgelerenteit instead of boekgeleerdheid (De Vries, Baie melk, p. 9); gelerentheid instead of geleerdheid (Son, 'Oom Sonnie', $19 / 4 / 2015$ )

- Diminutives ending on -entjie: karrentjie instead of karretjie (Small, KK, p.77); poppentiie < poppie (Otto-Sallies, Diekie, p. 25); traffic-ouentiie instead of traffic-outjie/traffic officer (Own recording); vrouentije instead of vroutjie (Own recording)

- Verbs:

- Verbs ending on $-e$ : beginne for begin (Small, KHKH, p. 49); worre < worde instead of word (Small, KHKH, p. 54); ytvinne instead of uitvind
(Otto-Sallies, Diekie, p. 71; Small, $K K$, p. 80)

- Verbs ending on $-t$ instead of $-n$ :

gaat instead of gaan (Small, $K H K H$, p. 20); gat instead of gaan (Small, $K H K H$, p. 60); siet instead of sien (Small, KHKH, p. 25); ytgat instead of uitgaan (Otto-Sallies, Diekie, p. 63)

- galoef ( $<$ geloof) as verb (Small, $K K$, p. 80)

- Adverbs:

- Adverbs ending on $-s$ : glads instead of glad (De Vries, Baie melk, p. 99); nogals instead of nogal (De Vries, Baie melk, p. 86); orals instead of oral (Snyders, Political Joke, p. 40); skoons instead of skoon (De Vries, Baie melk, p. 82; Snyders, Political Joke, p. 38)

- Other adverbs:

hoeka (Otto-Sallies, Diekie, p. 51); kammakastag instead of kamma (De Vries, Baie melk, p. 77; kammatjies instead of kamma (Small, KHKH, p. 62); langesaan instead of langsaan (Son, 'Oom Sonnie', 19/4/2015; Small, KHKH, p. 52)

- The indefinite numeral baieng instead of baie (Own recording)

- The pronoun form selwers instead of self:

Lat ons die einde liefs ma' selwers maak. (Small, KMK, p. 64)

11 Kotzé (1984) uses the concept “centralization” to describe the modification of the phonemes /o/ en /o/ to the central phoneme $/ \mathrm{o} /$. 
(p) Common sayings/interjections/ expressions in Kaaps

bos los $\{$ Give the secret away\}:

Nadat ons die twee bejaardes (...) gelawe het, het hulle bos gelos oor die swart kat-gedaante wat as beskermengel oor hulle gespeel het. (Son, 'Oom Sonnie', 19/6/2012)

gooi 'n lange \{leave $\}$ :

Vat daai stukkende BM en gooi 'n lange voor ek jou met die vuiste hit. (Son, 'Oom Sonnie', $17 / 4 / 2015)$

Iemand 'n kop aansit \{openly lie to someone; mislead\}:

Danny haal sy skouers op. 'Hulle het my ook 'n kop aangesit.' (Son, 'Oom Sonnie', 1/12/2014)

in die gesig vat \{insult; belittle\}:

'op elke plek, op elke pad word ons in onse gasig gevat' (Small, KMK, p. 70)

in die gesig spoeg \{insult; belittle\}:

'ma' aldag word in onse gasig gespoeg' (Small, KMK, p.79)

in die krop bêre \{remember\}:

'bêre dit in die krop!' (Small, KMK, p. 79)

in die ö̈/eyes sit \{embarrass\}:

Wat? Vi' jou, djy's dan altyd geroek, djy sal ons mos innie eyes sit. (OttoSallies, Diekie, p. 67)

Is toedoe $\{$ It's fine/okay! :

Moetie soe upset raak'ie, is toedoe, my broe' (Otto-Sallies, Diekie, p. 60)

kan gekom het is nice/attractive\}:

Daai's 'n baie nice couple, maa' die vrou kan nogal gekom het - sy't 'n mooi gesiggie soes dagliggie. (Koza, 'Stall'tjie', p. 84)

Lekka/lekke, djy! (exclamation of pleasure over something someone has said or done):
Lekka djy, oom Sonnie. Great stuff, oom Sonnie! (Son, 'Ditjies en Datjies', 8/7/2009)

Lekke djy, Antie Mona. Is reg, sit haar op haar plek. (Son, 'Ditjies en Datjies', 4/8/2010)

maak jou laat \{misleading oneself\}:

Ha, dink hy, daai sangoma maak haar laat. (...) Ja, hy sal sy ma 'n paar duisend (rand van sy wengeld) gee, maar die res is syne, en net syne alleen. (Son, 'Oom Sonnie', 2/11/2011)

'Djy maak djou laat as djy dink djy ga my soe easily vedala, bru,' gil die dude terwyl hy vinnig nader storm. (Son, 'Oom Sonnie', 21/2/2012)

min te wiet $\{$ not knowing\}:

Min te wiet dat dié visarend, wat eintlik 'n eagle is, 'n tamaai kabeldjou yt die water yt kan opsnap in 'n oegknip. (Son, 19/4/2015)

nie tyd vir slange hang nie \{immediately; without hesitation\}:

Daar is anyway nie tyd vir slange hang nie, want ons kat-heldin moet nog vir Quentin 'Gruwelik' Skippers vir sy gruweldade laat boet! (Son, 'Oom Sonnie', 19/6/2012)

op iemand se kerrie werk firritate someone\}:

Shorty is die complete opposite en maar 'n man van min woorde. Maar Langman én John S werk op sy kerrie. (Son, 'Oom Sonnie', 1/12/2014)

teen die $k a r(r e)$ skop \{refuse; protest\}:

Inwoners van South Road in Plumstead wat council-huise bewoon en binnekort op straat gaan sit oor 'n MyCiti- busroete, skop teen die karre. (Son, 3/2/2015)

Hy sê hy't 'n brief geskryf en gevra om terug te gaan na sy seksie 
toe, maar die onderhoof van die gevangenis het teen die kar geskop. (Son, 15/7/2011)

uit jou boom uit wees \{being crazy\}:

'Dy's yt jou boem yt!' het ek hom gesê.

'Djy's mal!' (Snyders, Voete op aarde)

vir 'n pop/apie vat \{underrate someone's intelligence; underestimate $\}$ :

'Djy,' sis Makro, 'djy het my vir 'n pop gevat. (...) Jy verdien om te sterf.' (Son, 'Oom Sonnie', 2/3/2012)

Sy het sy liefde vir 'n pop gevat. Hy sal dit nie só aanvaar nie. (Son, 'Oom Sonnie', 21/2/2012)

Hy sal staan en afkyk hoe haar rukkende liggaam nog die laaste bevele van die brei probeer gehoorsaam. Sy wou hom mos vir 'n apie vat. (Son, 'Oom Sonnie', 21/2/2012)

What kaain/kind met jou? \{ What is going on with you? / What do you have to say?\}:

Djy't gel' vi' pille, ma' djy't nooit gel' vi' twak'ie. What kaain met jou? (OttoSallies, Diekie, p. 11)

'What kind?' vra sy maat. (Son, 'Oom Sonnie', 8/6/2011)

The items listed in (k), (l), (m), (n), (o) and (p), represent the following tendencies regarding the use of lexical items and expressions in Kaaps:

i) In accordance with the dynamic nature of Kaaps, the lexical items in (k) differ spatio-temporally: items such as entjie (cigarette) and vertel (insult/tell off) have been in use for a long time, while items such as aweh, dydelik/duidelik, eish, gaga, skanghaka/ skanghagha, twaisfai and woelag are more recent.

ii) Some of the lexical items in (k) are, according to form, ordinary Afrikaans words that have undergone an expansion of meaning in Kaaps. Among these are items like afbene/afbiene (broke), afsak (say), bad (squander or serving prison sentence), dydelik/duidelik (undeniably good), gevaarlik (very), mol (in a hurry to get away) and ytstiek (turn up).

iii) Kaaps draws lexically from many spheres of usage. The lexical items and expressions in (1) and (p) (and the text data used as illustrative material) suggest that the Cape underworld (gangsterism, imprisonment, drug practices) is a fertile breeding ground especially for lexical innovations and new expressions. Accordingly, one can observe an underworld register as a prominent register distinction in Kaaps. Regarding the usage of lexical items and expressions, Kaaps, in accordance with Anastasia de Vries (2006), is metaphorically speaking a sponge that sucks up influences from speech codes such as gay, street, gang and prison language:

... like a sponge Kaaps sucks up influences from the gay, street and gang language, and Malay, Xhosa and Arabic elements emerge alongside a wealth of own innovations (...) While some of the expressions have a reasonable shelf life and are (can be) adapted from one generation to another, those linked to gang activities and prison life are rather shortlived.

Code influence from geographic spaces outside Cape Town and environs have also come into play. With Flaaitaal (Tsotsi Afrikaans), for example, Kaaps shares, among others, the lexical items listed in (m). 
iv) Kaaps is marked by a number of synonymic lexical items and expressions. To name police officials there is a choice between the items boere, gattas and mapoezas. Someone using the drug tik is a tikkop, tjoefkop or a tjoefer. A form of address for a friend can be bra/bla/broe(r)/bru or buddy or tjommie or pel/pêl/pellie. I should add: it is not unusual to use these forms of address for a girl (lady) friend as well. Something pleasing is in Kaaps either kwaai or skanghaka or woelag or kwaailappies. The expressions iemand 'n kop aansit and iemand vir 'n pop/apie vat are approximiately synonymous.

\section{The grammatical characteristics of Kaaps}

Typical grammatical (morphological and syntactic) phenomena in Kaaps are listed in $(\mathbf{q})$. The grammatical phenomena in (r) are found in the literary texts of S.V. Petersen, Adam Small and Elias P. Nel, among others, but seldom occur among contemporary speakers of Kaaps and in text in which contemporary Kaaps has been captured. These phenomena are thus considered to be obsolescent.

\section{(q) Grammatical phenomena in Kaaps}

- Morphological embedding of English loanwords:

- In combination with $g e$-: ge-ig $<$ ge-ignore (Snyders, Die Burger Landelik, 23/8/2001); ge-organise (Son, 'Oom Sonnie', 16/4/2015); ge-prepack (Son, 'Oom Sonnie', 9/4/2015); ge-trace (Son, 'Oom Sonnie', 15/4/2015)

- in combination with the diminutive morpheme:

boytjie (Own recording); stall'tjie (Koza, 'Stall'tjie', p. 79);
- as modifier components in hybrid compounds: entertainment-kamer (Son, 'Oom Sonnie', 10/4/2015); (Die Kaapse) gangland (Son, 'Oom Sonnie', 9/4/2015); security-mense (Son, 'Oom Sonnie', 8/4/2015); takeaway-boksie (Son, 'Oom Sonnie', 9/4/2015)

- as verb element in hybrid particle verbs:

ansain < sign on (Petersen, 'Rotjies'), opjôin < join up (Petersen, 'Rotjies'), ytgewear < worned out (Snyders, Die Burger Landelik, 10/5/2001)

- Duplication of plural morphemes:

- brasse/bra'se (instead of bra's): Aau, wat soo'te bra'se is julle? (Otto-Sallies, Diekie, p. 57)

My bra'se is ghrên laaities (OttoSallies, Diekie, p. 60)

My brasse het gesê omdat ek 'n juvenile is (...) sal ekkie straf kry nie. (Son, 'Oom Sonnie', $1 / 3 / 2015)$

- broerse (instead of broers):

Toe storm 'n paar van my broerse na buite. (Son, 'Oom Sonnie', 1/4/2010);

$\mathrm{Ek}$ is weer tussen my broerse. (Son, 'Oom Sonnie', 1/4/2010)

- ouense (instead of ouens)

$\mathrm{Ag}$ toe, ouense, stoppit, man. (Otto-Sallies, Diekie, p. 11)

- Plurals with -ens:

jongens (Son, 'Oom Sonnie', 13/11/2012); ouens (Otto-Sallies, Diekie, p. 11; Son, 'Oom Sonnie', 13/11/2012); vrouens (Trantraal, Rapport Weekliks, 10 January 2016, p. 15) 
- Diminutive forms with -entiie: karrentjie instead of karretjie (Small, $K K$, p.77); poppentiie instead of poppie (Otto-Sallies, Diekie, p. 25); traffic-ouentjie instead of trafficoutjie/verkeerskonstabel (Own recording); vrouentjie instead of vroutjie (Own recording)

- Word formation by curtailment:

- ig/ge-ig (< ignoreer, geïgnoreer; ignore, ignored)

Toe hulle my besware $i g$, kyk ek weg en gaan in 'n hewwie salk. (Snyders, Nursie)

Ek het alles maar ge-ig. As djy nie notisie van hulle glimlagterapie vat nie, dan affek dit jou nie. (Snyders, Die Burger Landelik, 23/8/2001)

As hy op 'n stasie of anner plekke advice in sy gebroke Duits vra, het hulle hom net soe ge-ig as wat hy Engels gepraat het. (Snyders, Die Burger, 6/2/2004)

- info ( $<$ information)

'Hoe weet jy al dié goed? Dis mos gevaarlike info, jong?’ vra André en staan op. (Son, 'Oom Sonnie', $14 / 4 / 2015)$

- nemma $\quad(<\quad$ nemmatjies $<$ netnoumaartjies) (Petersen, 'Bandeloos')

- Sarag/Saarag (< Saterdag)

Elke Saarag lieg djy vi' haar' dan gat julle matinee djol (OttoSallies, Diekie, p. 35)

Ek sê, ouense, Saraagaan' is ons mobile. (Otto-Sallies, Diekie, p. 65)

- Word formation by curtailment plus diminution:

- onnie(s) (< onderwyser(s)):

Maar vir eers moet daai onnie en sy vriend wag. (Son, 'Oom Sonnie', 16/4/2015)

'Sak jul koppe,' skreeu hy aan die twee onnies terwyl hy wegtrek. (Son, 'Oom Sonnie', 15/4/2015)

- $\quad$ smokkie(s) (< smokkelhuise):

Dodelike geveg in smokkie lei tot die dood ... (Son, 'Oom Sonnie', 6/6/2011)

Daar's nie onnodige gunskietery nie. Niks gangsters op die straathoeke of lawaaierige smokkies by elke tweede huis nie. (Son, 'Oom Sonnie', 28/1/2013)

- Addition of a comparative suffix:

- to (Afrikaans and English) adjectives:

bieterder instead of beter (De Vries, Baie melk, p. 86); cheaperer instead of cheaper (Snyders, Political Joke, p. 37); ergerer instead of erger (Snyders, Political Joke, p. 38); ha'terre (< ha'terrer) instead of harder) (Otto-Sallies, Diekie, p. 73); langere (< langerer) instead of langer (Small, $K K$ ); seiferer instead of safer (Snyders, Political Joke, p. 32); slimmere (< slimmerer) instead of slimmer (Otto-Sallies, Diekie, p. 39); swarterer instead of swarter (De Vries, Baie melk, p. 76); vinnagere (<vinnagerer < vinniger) (OttoSallies, Diekie, p. 74)

- to indefinite numerals: mere $(<$ merer < meerder < meer) (Otto-Sallies, Diekie, p. 27); minere/minnere ( $<$ minnerer/ minderer $<$ minder) (Small, $K K$; Otto-Sallies, Diekie, p. 32)

- to locative adverbs:

Hy woon 'n bietjie afferer/after (instead of verder af); Dis nog opper/opperer. (Kotzé 1984) 
- Non-emphatic adjectival declension: die arme families in die straat (OttoSallies, Diekie, p. 17); die korte, korte wit skirtjie (De Vries, Baie melk, p. 28); mooie bossies narsings! (Small, KK, p. 75); die skone bier (Snyders, Political Joke, p. 3); soete kinners (Small, KMK, p. 70); 'n stoute kind (Otto-Sallies, Diekie, p. 62); warme koesiestes (De Vries, Baie melk, p. 98)

- The use of lexical indirect vocatives (instead of $j y / u$ ):

Oemie, ek het vi' Oemie regtag gemis, die hys wassie dieselle sonne' Oemie nie. Kom ek help vi' Oemie kame' toe. (Otto-Sallies, Diekie, p. 15)

Willie ou (< wil die ou) nie saam gaan nie? (Nel, Verneukpan, p. 143)

- Regularisation of verb forms:

- het gekan instead of kon: So hy't nie gekan wêk nie (Small, $K K$, p. 81)

- het wil ... het (instead of wou): Toe't sy weer 'n anner werk wil gedoen het. (Small, KHKH, p. 54)

- het instead of hê: Sukke vrinne lyk ek djy moet het. (Otto-Sallies, Diekie, p. 61)

- sal instead of sou: Wakes, ..., sal jy gecheck et toe jy klein was jy sal ooit te veel chips kan iet? (Trantraal, Rapport Weekliks, 10 January 2016, p. 15)

- End-positioning of, inter alia, time and place expressions and objects:

Ek ga'n mos kerrie maak vanaand. (Koza, 'Stall'tjie', p.79)

Hulle't biesagheite gebou daar. (Small, KHKH, p. 67)
Die Here het aan my gebring sy wonnerwerke. (Small, KHKH, p.33)

- Placing of the definite article in front of place and street names: innie Delft (Son, 'Oom Sonnie', 12/8/2012), innie Lavis (Trantraal, Rapport Weekliks, 10 January 2016, p. 15), op die Bellville-stasie (Son, 'Oom Sonnie', 5/4/2015), vannie Makka (Otto-Sallies, Diekie, p. 14); innie Blomstraat (Small, KKH, p. 59), innie End Road (Trantraal, Rapport Weekliks, 10 January 2016, p. 15)

- Placing of the definite article in front of uncountable substantives (mass nouns):

Hy't die tiebie gahad. (Small, $K K H$, p. 62)

Hy's baie goed moerie $(<$ moet die $<$ met die) masiek. (Small, KKH, p. 61)

Hy's dood want hytie flu $(<$ hy het die flu) gehad (Own recording)

- The use of the prenominal construction ' $n$ nog ' $n$ (instead of $n o g$ 'n) according to the rhythm pattern of the English item 'another': 'n nog 'n kan (Snyders, Political Joke, p. 50); 'n nog 'n rand (Snyders, Political Joke, p. 19); 'n nog ' $n$ swarte (Snyders, Political Joke, p. 67)

- Use of it (instead of dit) and is (instead of dis) at the beginning of a sentence:

It gaan bars! (Small, KHKH, p. 45)

It wasse goeie jaa vi ôs. (Trantraal, Rapport Weekliks, 10 January 2016, p. 15)

Is sieker weer ou Boela se vis. (Small, KHKH, p. 42) 
Is eintlik al riede hoekom ôs so lekke kan sit en movies kyk. (Trantraal, Rapport Weekliks, 10 January 2016, p. 15)

- Use of the possessive pronoun onse (instead of ons) in attributive position:

onse baby (Otto-Sallies, Diekie, p. 79); ôsse (< onse) kinners (Son, 'Oom Sonnie', 21/6/2010); onse koppe (Snyders, Die Burger Landelik, 24/5/2001); onse mense (De Vries, Baie melk, p. 56); onse way van doen (De Vries, Baie melk, p. 56)

- Repetition of prepositions (in, uit) with dynamic and static verbs:

So daai tyd ons lat hom toe innie kaap in bly ... (Small, KKH, p. 59)

O's lies yt Oepenbaring yt (De Vries, Baie melk, p. 34)

Min te wiet dat dié visarend, wat eintlik 'n eagle is, 'n tamaai kabeldjou $y t$ die water $y t$ kan opsnap in 'n oegknip. (Son, 19/4/2015)

- Use of daai/dai instead of the pronoun dit:

Daais (< Daai is) Kanna sy stem, meneer. (Small, KKH, p. 63)

Got hoe wiet djy dáái, hi? (Small, $K K$, p. 75)

Ôs het dai gedoen ..., ôs het ôsself $d a i$ gelee. (Trantraal, Rapport Weekliks, 10 January 2016, p. 15)

- Use of the quantor almal (instead of $a l)$ as collective marker:

... sê almal die mense (Small, $K M K$, p. 53)

'Kom gee almal julle geld en maak leeg julle sakke!' skree die jong. (Son, 'Oom Sonnie', 5/4/2015)
- Use of die preposition vir in front of a human object:

Hy't vir Kietie liefgehad. (Small, KHKH, p. 51)

Toe sy vir Koelie sien, gryp hulle mekaar (Otto-Sallies, Diekie, p. 78)

'Nou hoeko' het Mammie vi' my lat slaap!' skree sy. 'Die dokte' het vi' jou 'n injection gegie, Ice ...' (OttoSallies, Diekie, p. 80)

\section{(r) Obsolescent grammatical} phenomena in Kaaps

- Repetition of the subject in pronominal form:

Kanna $h y$ 't nou baie geld gemaak. (Small, KKH, p. 60)

Klein Kytie sy't oek ma gahyl. (Small, KKH, p. 61)

Melancholy, it vat my ienagge tyd even innie mirrel vanne action film. (Trantraal, Rapport Weekliks, 10 January 2016, p. 15)

- Use of a possessive pronoun instead of $s e$ :

Kanna sy stem (Small, KKH, p. 63); die ou mêrim haar naam (Small, $K K H$, p. 60)

- Verbal hendiadys with te (instead of en), as in Standard Dutch:

Dan staan jy daar te tjank. (Kotzé 1984)

Daar sit ek te praat met hulle. (Kotzé 1984)

- Use of $s e$ in combination with the possessive pronouns $u$ and julle and with time expressions like nou and eers:

U se broer Piet / julle se vriende / nou se Maleiers (Kotzé 1984) 
- Nominalisation of the indefinite pronouns iets and iemand:

Hy's 'n mens wat doen goeie ietse. (Kotzé 1984)

... unless is 'n sjeg of 'n iemand (Kotzé 1984)

\section{Concurrence between Kaaps and Standard Afrikaans}

As colloquial variety of Afrikaans, Kaaps (like other forms of colloquial Afrikaans) interacts with Standard Afrikaans, therefore Standard Afrikaans influences Kaaps and Kaaps (at least potentially) serves as feeder source for Standard Afrikaans. This interaction flows from Kaaps speakers' exposure to Standard Afrikaans. As indicated in (s), Kaaps is characterised linguistically by a concurrence between typical Kaaps and standardised variants.

\section{(s) Interchange between Kaaps and standardised variants}

- Affricatisation of /j/ vs. no affricatisation:

- As djy vi' my iets wil sê, dan sê $d j y$ vi' my iets bietere (Otto-Sallies, Diekie, p. 63)

$\mathrm{X}$

Ek sê vi' jou dié because ek is jou vrin' (Otto-Sallies, Diekie, p. 63)

- djaar (Small, KHKH, p. 53)

$\mathrm{x}$

jaar (Small, KHKH, p. 13)

- Post-vowel /r/-omission vs. retention thereof:

As djulle vroumense $e e^{\prime} s$ aan die skinne' raak, kan 'n traffic cop djulle nie kee' nie. Maar sê ee's, waa' issie toilet? (Koza, 'Stall'tjie', p. 82) $\mathrm{x}$

Ek het vir hulle mooi geverdydelik (Small, KHKH, p. 55)

- Lowering vs. retention of schwa: afgaleer (Small, $K M K, \quad$ p. 17); biesagheid (Koza, 'Stall'tjie', p. 81); haastag (Koza, 'Stall'tjie', p. 79)

$\mathrm{X}$

gekoekte kos (Son, 'Oom Sonnie', 1/3/2015); miskien (Small, KMK, p. 19)

- Raising vs. no raising of vowels:

uitstiek (Son, 'Oom Sonnie', 12/11/2012)

$\mathrm{x}$

uitgesteek (Son, 'Oom Sonnie', $30 / 1 / 2013$ )

- Morphological curtailment of items vs. no curtailment:

Saarag (Otto-Sallies, Diekie, p. 35) $\mathrm{x}$ Saterdag (Son, 'Oom Sonnie', 12/8/2012);

onnies (Son, 'Oom Sonnie', 9/4/2015) $\mathrm{x}$ onderwysers (Son, 'Oom Sonnie', 9/4/2015)

- Place names preceded by die vs. place names not preceded by die:

'innie Delft' (Son, 'Oom Sonnie', 12/8/2012), 'vannie Makka' (OttoSallies, Diekie, p. 14) x

'in Mitchellsplein' (Koza, 'Stall'tjie', p. 79), 'yt Windermere' (Small, $K M K$, p. 23)

\section{KAAPS AS HETEROGENEOUS LANGUAGE FORM}

Kaaps does not manifest as a variety with uniform use; in fact, Kaaps is essentially a heterogeneous variety of 
Afrikaans. Firstly, the usage of Kaaps display idiolectic differences. With one specific speaker, phonological markers are dominant, while the Kaaps of another speaker is more specifically marked lexically or grammatically. As Anastasia de Vries (2006) indicates, these idiolectic differences are also culturally determined: "The kind of Kaaps you speak is (...) a roadmap of the cultures and subcultures you are exposed to.' There are also apparent social group or register differences (including Kaaps among Muslims vs. Kaaps among Christians vs. the register of the Cape underworld), economic class differences (Kaaps among the working class vs. Kaaps among the middle class) as well as regional differences (including Kaaps of the Bo-Kaap vs. Kaaps in Mitchells Plain on the Cape Flats), and so on. Further empiricial research should confirm that the variation in Kaaps is subject to the determining influence of variables such as religious orientation, age, exposure to Standard Afrikaans and degree of literacy. ${ }^{12}$ Ponelis's use of the plural labelling 'the varieties of the Cape Flats' (Ponelis 1998:14), 'the subvarieties of Cape Town and the Peninsula' (Ponelis 1998:15) and 'vernacular subvarieties (of Southwestern Afrikaans)' (Ponelis 1998:15) to characterise Kaaps, or rather Cape Vernacular Afrikaans, matches the heterogeneous nature of this language form.

\section{THE SOCIAL}

\section{EMBEDDEDNESS AND}

CONTEXTUAL USES OF

\section{KAAPS}

Some speakers use Kaaps as primary language code, thus as mother tongue, in nearly all situations. For others (and probably most), Kaaps is currently a secondary or occasional code, i.e. a communication form reserved for informal exchange. That said, Kaaps cannot be pinned down within a geographically defined space, and several of its users are in command of other languages codes (such as English or another Afrikaans variety) as well. In conjunction with the dispersion, mobility, literacy levels and social differences of its users, Kaaps features in the multilingual set-up of the Cape Peninsula in interaction with other language codes, and in a broad code-switching practice, linguistic aspects are exchanged daily in various locations and social spaces. For its users Kaaps is thus a language code that can either be stored or taken out when needed, according to the norm of situational suitability.

Kaaps is a functionally adequate language code. It has long operated as follows: as a religion code among a significant number of Cape Muslims and Christians; as singing code in genres of Cape Malay folk songs, such as

12 In empirical research on Kaaps, the following could serve as hypotheses:

- Lexical items linked to the Islamic religion have a higher frequency in Bo-Kaap Afrikaans than among Kaaps speakers who practice the Christian religion, because Bo-Kaap Afrikaans is historically interwoven with the cultural life of the Cape Muslim community.

- Prosodic Afrikaansification of lexical items and expressions derived from English (back > bek, post office $>$ pous-offies, Don't you worry $>$ Dount joe warrie) and colloquial etymological distortion of English lexical items (expensive > expensis, Western Province > Westenpromis) occur especially among Kaaps speakers with a relatively low degree of schooling.

- Lexical items and grammatical phenomena which are remains of older language layers, are more frequent among older speakers than younger speakers.

- The alternation between typical Kaaps and standardized variants (e.g. djy x jy, boem x boom, trammakassie $\mathrm{x}$ dankie) is more frequent in the idiolects of Kaaps speakers exposed to formal schooling. 
moppies and ghoema songs; in musical performances, such as District Six, The Musical, and Kat and the Kings; in Hip Hop productions, as well as in the song and rap productions of music groups, such as 'Brasse vannie Kaap'.

Kaaps is also used in the business world as advertising medium. The product specifications on advertising boards and windows of businesses, as well as the (often poetic) jingles of hawkers, and of flower and fish sellers, serve as proof:

- Lekke' warme worsies! (Own recording)

- Mêrim, mêrim worrels, een hang los, twie ran' 'n bos! (Own recording)

- Rooikraans (instead of rooikrans) (Own recording)

- 'Ek sê, Mêrrem! Koep 'n kool vir Master se tool! Tamatie virrie paartie! Avokadopere maak die hare me're! Die ywe gie die flavour! Kyk maa' wee' wat djou ma'g makee! Lekka Golden Delicious apples. Buy them for your rimples en smile met 'n dimple! Een byt ennie hiening spyt - die bye is my getye!' (Koza, 'Stall'tjie', p. 79)

- 'Hie's djou lekke snoeke, mammieee!' skreeu die snoekverkopertjie dit uit op die Bellville-stasie. 'Die prys is reg en die baas is weg! Dis Easter, mense met julle ronne pense!' (...) 'Net reg virrie piekel fish, vra ma' virrie mies!' (Son, 'Oom Sonnie', $5 / 4 / 2015$ )

Although Kaaps is primarily a spoken language variety (thus a form of 'Praatafrikaans'), it has over the years also been utilised as written medium in journalism (as in Peter Snyders' column
'Kopstukke' in Die Burger a few years ago, and currently also in the Cape newspaper Son), and also in various literary genres (poetry, drama, prose) with figures such as S.V. Petersen, Adam Small and Peter Snyders as prominent exponents. These writers and others have contributed to establishing a codified register that Ernst Kotzé (2001:106-107) calls the 'Literary Cape Vernacular'. According to Kotzé, this code aims to reflect informal spoken discourse by adapting the orthography in order to capture the typical pronunciation of Kaaps.

The application of Kaaps as written code goes back a long way. Kaaps was engaged in one of the first Afrikaans codifying system, namely the Arabic Afrikaans writing tradition. This tradition had its origin in the first half of the 19th century in Cape Muslim schools and remained in use until the middle of the 20th century. Accordingly, Cape Muslim Afrikaans had, for religious purposes, been put into writing successfully by means of an adapted version of the Arabic alphabet (Botha 1989:135; Davids 1991; Davids 2011). ${ }^{13}$ Further, at the beginning of the twentieth century, specifically between 1909 en 1922, Kaaps was utilised as written medium in 'Straatpraatjes', a satirical column in $A P O$, mouthpiece of the African People's Organization, which was edited by one 'Piet Uithalder' who was presumably Dr Abdulla Abdurahman (Adhikari 1996; Ponelis 1996).

\section{KAAPS IN LINGUA- POLITICAL PERSPECTIVE}

Ponelis (1998:3, 1999a) views Afrikaans, metaphorically, as a family of varieties. As member of the Afrikaans family, Kaaps 
was however despised and suppressed as colloquial code for most of its existence, thus stigmatised and marginalised. The labelling of Kaaps in literary works as 'Coloured Afrikaans' (Retief 1964:168), 'the crooked language of the Coloured' (Weideman 1964:210), 'Coloured patois' (Dekker 1970:276-77), 'Gamat language' (Small 1961:9) and 'Capey' (Small 1961:9) is actual confirmation of this. The notions of 'Gamat language' and 'Capey', specifically, are linked with 'patronising scorn' by Adam Small (1997:223).

Since the beginning of the 20th century until the height of Apartheid, Kaaps was purposely circumvented in the standardisation of Afrikaans, and sidestepped in the linguistic description and tuition of Afrikaans. As a reflection of Afrikaner nationalism, these forms of marginalisation not only promoted the stigmatisation of Kaaps, but also resulted in Kaaps being sensed by its own speakers as being an inferior Afrikaans.

Choosing Eastern Afrikaans (also known as Eastern border Afrikaans), instead of Kaaps or Orange River Afrikaans, as basis for the standardisation of Afrikaans (as indicated by Van Rensburg and Ponelis), implicately amounted to a refusal to build on one of the first Afrikaans codifying systems, namely the Arabic Afrikaans writing tradition of the 18th and 19th century. In this witing tradition, as stated before, Cape Muslim Afrikaans, a prominent subvariety of Kaaps, had been successfully put into writing by means of an adapted version of the Arabic alphabet. One realizes that if the Arabic writing tradition had been taken into account or as point of departure at the beginning of the twentieth century, Standard Afrikaans may today have had a very different character, more specifically one strongly tinted with Kaaps.

\section{FUTURE PERSPECTIVE ON KAAPS}

What are the challenges regarding Kaaps? What is the road forward for Kaaps and for Afrikaans in general?

One would hope that the linguistic and social nature of Kaaps, as well as its scope of usage will in future be researched anew and continuously, and that the findings of such research will be recorded in dissertations, academic publications and dictionaries. One investigation theme could be to ascertain to what extent Kaaps, or aspects thereof, have infiltrated other colloquial codes and been absorbed by them. Relevant codes are, among others, the informal colloquial speech of standard language speakers of Afrikaans and English, the lingua franca in multilingual informal settlements in the Cape Peninsula as well as cyberspace codes like email and SMS language. One could also investigate how Kaaps figures in cities abroad like London and Perth, where a significant number of Cape-born (e)migrants are gathered. Personally, I would like to participate in producing linguistic works in which Kaaps is accommodated along with other Afrikaans colloquial varieties and Standard Afrikaans into one descriptive framework. Such polylectic language description should indeed confirm the kinship of Afrikaans varieties which Ponelis $(1998: 3$; 1999a) has emphasized. There is also an appeal to correct the historic process of defining Afrikaans colour varieties, and Kaaps in particular, outside of the standardisation of Afrikaans.

There is also an appeal to ensure a historical rectification regarding the disregard of Afrikaans colour varieties, particularly Kaaps, as feeder sources for Standard Afrikaans. In the words of Le Cordeur (2010): 
If Kaaps Afrikaans (Muslim Afrikaans) had the largest number of speakers (more than 63000 in 1808), why did we allow our language (i.e. Kaaps - FSH) to take on, and to continue to take on, a subservient role?

Two choices are relevant in this regard (Hendricks 2011:213). One option would be to abandon the current standard variety and utilise one of the historically marginalised regional dialects, i.e. either Southwestern Afrikaans (including Kaaps as one of the key subvarieties) or Orange River Afrikaans, as new standardisation base. $^{14} \mathrm{~A}$ second option would be to reform the current standard variety in an evolutionary way, to recreate it by continually sifting it to retain that which is considered relevant, and to increasingly utilise Southwestern Afrikaans (especially Kaaps as subvariety) and Orange River Afrikaans as sources of supply. I would argue that the first option (as the seemingly politically correct one) might be too radical and that it could lead to the implosion of the Afrikaans language at large. The future of Afrikaans, in my view, lies in the reformation option, supported by inclusive regenerative socio-political forces.
For Kaaps to flourish as fully-fledged communication code alongside Standard Afrikaans, on the one hand, and to be considered a fertile productive supply source for the standard variety, on the other hand, it must be freed from its state of marginalisation and stigmatisation. A number of measures, like those specified by Hendricks (2011:113-114, 2012a:53$60)$, could be considered for this purpose.

Of cardinal importance is a paradigm shift, and a change of attitude, regarding our view of the relationship between the standard variety and the colloquial varieties of Afrikaans. The customary hierarchical perspective of variety diversity should make way for an egalitarian (or equal-level) perspective, according to which Standard Afrikaans and all colloquial varieties linked to it organically, are considered to be, in principle, equivalent codes of communication. A second measure would be to encourage free use of Kaaps and other colour varieties, in the private domain, but increasingly also as speaking and/or writing code in those public spheres of usage traditionally regarded as the domain of the standard variety: churches and mosques, courts of law, journalism, radio, television, conference podia, and the like (Combrink, 1998). In addition,

\footnotetext{
14 In the 1990s already, Ponelis raised the possibility of bringing about this rectification. The dialect Southwestern Afrikaans is central to his reflection on the early twentieth century standardisation of Afrikaans and his thoughts on the possibility to restandardise Standard Afrikaans. Ponelis (1998:64) is of opinion that early twentieth century Southwestern Afrikaans must have been the most suitable candidate to be chosen as the basis for standardising Afrikaans, because it is the dialect of the Afrikaans language's region of origin, and also a dialect with a strong urban core (Cape Town and the Boland) and a large number of speakers. He is also of the opinion that, if the Patriot movement at the end of the 19th century had had greater impetus, the southwestern dialect could have been the basis of Standard Afrikaans. Ponelis (1998:68) also considered the possibility of reforming Standard Afrikaans in order to counteract the increasing diglossia between Standard Afrikaans and colloquial Afrikaans. According to him, a possible remedy for this gulf would be to change the dialectical basis of Afrikaans by restandardising Standard Afrikaans on, for example, the basis of the southwestern (Cape) variety. The Kaapsification ("verkaapsing") of the culture language would then have the advantage that the new Standard Afrikaans becomes more accessible to a large number of users.
} 
there is an appeal to poets, prose writers and playwrights to continue with the practice of utilising, as literary medium, the colour and other colloquial varieties alongside the standard variety. As a third measure, an adjustment regarding language tuition can be considered. Afrikaans language tuition at school level should be increasingly instrumental in destigmatising colloquial varieties by maintaining a fine balance between mastering of the standard variety and recognising the usage validity of spoken language varieties. To lead learners to the insight that alternate usage of the standard variety and colloquical varieties (like Kaaps) is subject to the norm of contextual suitability, should be a primary outcome of language tuition.

What will be to the advantage of Afrikaans is an inclusive, more flexible, expansive standard variety, fed by the full spectrum of colloquial varieties, particularly Kaaps and other colour varieties. In my view, this is what should be understood under the concept 'democratization of Afrikaans'. The realization of this ideal will ensure that Standard Afrikaans can indeed feature as unifying variety in service of all groupings who associate themselves with Afrikaans.

\section{CONCLUDING REMARKS}

The Language Commission of the South African Academy for Science and Arts, on which I have served since 2010, has already for quite some time been committed to, and has already started with, the inclusion in the Afrikaanse Woordelys en Spelreëls of lexical items derived from Kaaps, especially, but also from Orange River Afrikaans (Taalkommissie 2009; Germishuys 2015). A significant number of items from Kaaps have also been included in the sixth edition of the Handwoordeboek van Afrikaans taal (HAT) (Luther et al. 2015). These initiatives signify a commitment to broaden the standard variety of Afrikaans by the inclusion of variants from the historically marginalised forms of Afrikaans.

A logical, and in my view unavoidable, additional initiative would be to speedily establish an Afrikaans grammar model which will reveal a polylectic perspective on Afrikaans and in which the rich linguistic systematics reflected in Kaaps will also be accounted for.

Kaaps is, as indicated, inextricably part of Afrikaans, and we are called upon to thoroughly take this into account, normatively as well as linguistically, on the road ahead.

\section{REFERENCES $^{15}$}

Adhikari, Mohammed (ed.). 1996.

Straatpraatjes. Language, Politics and

Popular Culture in Cape Town, 1909-1922.

Pretoria: Van Schaik.

Adhikari, Mohammed. 1996. Coloured identity and the politics of language: The sociopolitical context of Piet Uithalder's 'Straatpraatjies' column. In Adhikari, Mohammed (ed.) 1996, p. 1-17.

Anon. (n.d.) a Cape Town History - A Tourist Guide: Bo-Kaap. http://capetownhistory. co.za/?page_id $=236$

Anon. (n.d.) b Cape Town History - A Tourist Guide: District Six. ttp://capetownhistory. co.za/?page_id $=238$

Blommaert, Jan, Ben Rampton and Massimiliano Spotti. 2011. Language and Superdiversities. Diversities, 13 (2): $1-21$.

Botha, Theunis J.R. (ed.). 1989a. Inleiding tot die Afrikaanse Taalkunde. Second, revised edition. Pretoria: Academica.

Botha, Theunis J.R. 1989b. Afrikaans sedert die negentiende eeu. In Botha, Theuns J.R. (ed.), p. 127-154. 
Carstens, Adelia and Heinrich Grebe (eds.). 2001. Taallandskap: Huldigingsbundel vir Christo van Rensburg. Pretoria: Van Schaik.

Carstens, Wannie A.M. 2003. Norme vir Afrikaans: Enkele riglyne by die gebruik van Afrikaans. Pretoria/Cape Town: Academica.

Combrink, Johan. 1998. Engels as bron van Afrikaans: of Omgangsafrikaans is hoofsaaklik van Engelse herkoms. http:/www. und.ac.za/und/ling/archive/comb-01.html [accessed 20 October 2004].

Davids, Achmat. 1987. The role of Afrikaans in the history of the Cape Muslim community. In Du Plessis and Du Plessis (eds.) 1987, p. 37-59. . 1991. The Afrikaans of the Cape Muslims from 1815 to 1915: a socio-linguistic study. 2011. The Afrikaans of the Cape Muslims from 1815 to 1915. Unpublished M.A. dissertation, University of Natal. Hein Willemse and Suleman E. Dangor (eds.). Pretoria: Protea Book House.

Dekker, Gerrit. 1970. Afrikaanse literatuurgeskiedenis. Cape Town/ BloemfonteIn: Nasou.

De Vries, Anastasia. 2006. Kaaps, taal van my HART. Perspektief, Rapport, 23 July 2006.

Du Plessis, Hans. 1987. Variasietaalkunde. Pretoria: Serva Publishers.

Du Plessis, Hans and Theo du Plessis (eds.). 1987. Afrikaans en Taalpolitiek: 15 opstelle. Pretoria: HAUM.

February, Vernie. 1994. Taal en identiteit: Afrikaans en Nederlands. Cape Town: Tafelberg.

Gerwel, G.J. (Jakes) 2012. Language and silence: persoonlike herinneringe aan Adam Small, die digter-intellektueel. Tydskrif vir Letterkunde 49(1): 15-18.

Germishuys, George. Kwaailappies, my bra, ons is groos Afrikaans: Afrikaanse Woordelys en Spelreëls word hersien. http://www.netwerk24.com/Nuus/ Kwaailappies-my-bra-ons-is-groosAfrikaans-20150420
Hendricks, Frank. 2010. Op die taalspore van S.V. Petersen: 'n blik op sy verrekening van taaldiversiteit. Tydskrif vir Nederlands en Afrikaans (TNEA): 17(2): 21-37.

. 2011. Hoe gemaak met die ánder Afrikaans? Pomp 12: 111-114. . 2012a. Die potensiële nut van 'n gelykevlak-perspektief op die variëteite van Afrikaans. In Prah (ed.). 2012. CASAS Book Series no. 88: 44-63. .2012b. Om die miskende te laat ken - 'n blik op Adam Small se literêre verrekening van Kaaps. Tydskrif vir Letterkunde 49(1): 95-114. . 2014. Met Ponelis op die spoor van Praatafrikaans (Ponelis memorial lecture, delivered on 24 July 2014).

Hendricks, Frank S. 1978. 'n Sinchroniesdiachroniese studie van die taalgebruik in die drama KANNA HY KÔ HYSTOE van Adam Small. Unpublished M.A. dissertation, University of the Western Cape. . 1996. Kaaps: afwisseling met Engels is spontaan." Afrikaans Vandag, September 1996: 8-9.

Karaan, Munadia. 2009. Afrikaans en die Kaapse Moslem-gemeenskap vandag. (A contribution delivered during the Roots conference at UWC, 22-23 September 2009).

Klopper, Rembrandt Marius. 1983. Kaapse Afrikaans. Unpublished D.Litt. dissertation, University of Pretoria.

Kotzé, Ernst F. 1984. Afrikaans in die Maleierbuurt: 'n Diachroniese Perspektief. Tydskrif vir Geesteswetenskappe 24(1): 41-73. . 2001. 'n Perspektief op diachroniese prosesse in Afrikaans. In Carstens, Adelia and Grebe, Heinrich (eds.). 2001. p. 103-111. . 2010. Destandaardisasie en herstandaardisasie. Gelyklopende prosesse in die nuwe Suid-Afrika? In Van der Wal and Franken (eds.) 2010, p. 153-71. . 2012. Standaardisering en 'mainstreaming' - die kloutjie by die oor.

15 Please note that some authors only use their initials and surnames for all publications 
In Prah (ed.). 2012. CASAS Book Series no. 88: 30-43.

Le Cordeur, Michael. 2010. Bal die vuis vir Kaaps. Die Burger, 17 March 2010, p. 11.

. 2011. Die variëteite van Afrikaans as draers van identiteit - 'n Sosiokulturele perspektief / The varieties of Afrikaans as carriers of identity - A socio-cultural perspective. Tydskrif vir Geesteswetenskappe, Vol. 51(4): 758-777.

Luther, Jana, Fred Pheiffer and Rufus H. Gouws (eds.). 2015. HAT. Handwoordeboek van die Afrikaanse Taal. Sixth edition. Cape Town: Pearson.

Müller, Dalene and Sebastian Pistor. 2011. Skryf Afrikaans van A tot Z: die essensiële gids vir taalgebruikers. Cape Town: Pharos.

Nienaber, G.S. 1952. Benaminge van ons taal. In Serfontein, Martin J. (compiler): 1952: 62-68.

Pheiffer, R.H. 1996. 'Straatpraatjes' - 'n vroeë voorbeeld van Alternatiewe Afrikaans. In Adhikari, Mohammed (ed.) 1996, p. 141-162.

Ponelis, Fritz A. 1987. Die eenheid van die Afrikaanse Taalgemeenskap. In Du Plessis and Du Plessis (eds.) 1987, p. 3-15. . 1989a. Die aard en die bestudering van die Afrikaanse taal. In Botha (ed.) 1989, p. 9-37.

. 1989b. "Ontwikkeling van klusters op sluitklanke in Afrikaans". Suid-Afrikaanse Tydskrif vir Taalkunde 7(1): 1-5. . 1994. Standaardafrikaans in oorgang. In February, V. 1994, p. 106-128. . 1996. Codes in contradiction. The sociolinguistics of 'Straatpraatjes'. In Adhikari, Mohammed (ed.) 1996, p. 129-140. 1998. Standaardafrikaans en die Afrikaanse Taalfamilie. Stellenbosch: University of Stellenbosch. Annals 98(1): 1-80. . 1999a. Die oorspronge van Afrikaans. http://www.archive.org/ stream/rosettaproject_afr_detail-6/ rosettaproject_afr_detail-6_djvu.txt (accessed 25 May 2014). 1999b. Hesseling: 'n eeu later. Literator 20(1): 1-13. (http://literator.org.za/index. php/literator/article/viewFile/441/602) (accessed 25 May 2014). . 2009. Die taal wat ons praat, $B y$, Saturday 19/12/2009.

Prah, Kwesi Kwaa (ed.). 2012. Veelkantiger Afrikaans: Streeksvariëteite in die standaardvorming. CASAS Book Series no. 88.

Retief, P.J.P. Die Kleurling en die Afrikaanse Letterkunde. In Erica Theron (ed.) 1964.

Serfontein, Martin J. (compiler). 1952. Kristalle: gekeurde essays. Johannesburg: Afrikaanse Pers-Boekhandel.

Small, Adam. 1973 [1961]. Kitaar my kruis. Cape Town/Pretoria: Hollandsch Afrikaansche Uitgevers Maatschappij. . 1997. "Stryd, op my manier. In Willemse et al. 1997: 218-225.

Taalkommissie (van die Suid-Afrikaanse Akademie vir Wetenskap en Kuns). 2009. Afrikaanse Woordelys en Spelreëls (10th comprehensive revised edition). Cape Town: Pharos.

Theron, Erica (ed.). 1964. Die kleurlingbevolking van Suid-Afrika. Stellenbosch/Grahamstown: University Publishers.

Van der Wal, Marijke and Eep Francken (eds.). 2010. Standaardtalen in beweging. Amsterdam: Stichting Neerlandistiek VU.

Van Rensburg, Christo (ed.). 1997. Afrikaans in Afrika. Pretoria: Van Schaik.

Van Rensburg, M.C.J. 1989. Soorte Afrikaans. In Botha, T.J.R. (ed.). 1989, p. 436-467.

Weideman, Clemenso. 1964. Die bydrae van die Kleurlinge tot die Afrikaanse Letterkunde. Unpublished M.A. dissertation, University of the Witwatersrand.

Willemse, Hein, Marion Hattingh, Steward van Wyk and Pieter Conradie. 1997. Die reis na Paternoster. 'n Verslag van die tweede swart Afrikaanse skrywersimposium gehou op Paternoster vanaf 29 September tot 1 Oktober 1995. Bellville: University of the Western Cape. 


\section{Database}

- Linguistic and normative sources for Kaaps data:

- Kotzé, Ernst F. 1984. Afrikaans in die Maleierbuurt: 'n Diachroniese Perspektief. Tydskrif vir Geesteswetenskappe 24(1): 41-73. [Text reference: Kotzé 1984]

- Müller, Dalene and Sebastian Pistor. 2011. Skryf Afrikaans van A tot Z: die essensiële gids vir taalgebruikers. Cape Town: Pharos. (pp. 36-40) [Text reference: Müller and Pistor 2011]

- Literary texts from which linguistic aspects of Kaaps were obtained:

- A text by De Vries, Anastasia: De Vries, Anastasia. 2010. Baie melk en twie sykers. Protea: Protea Book House. [Text reference: De Vries, Baie melk]

- A text by Koza, Leonard: Koza, Leonard. 2002. Abdullah en Miena se stall'tjie. In Die Afrikaanse Skywersvereniging. 2002. Mense is mense. Pretoria: LAPA. [Text reference: Koza, 'Stall'jie']

- A text by Nel, Elias P.:

Nel, E.P. 1998. Iets goeds uit Verneukpan? Cape Town: Tafelberg. [Text reference: Nel, Verneukpan]

- A text by Otto-Sallies, Zulfah: Otto-Sallies, Zulfah. 2002 [1997]. DIEKIE vannie Bo-Kaap. Cape Town: Tafelberg. [Text reference: Otto-Sallies, Diekie]

- Texts by Petersen, S.V.: Petersen, S.V. 1943. "Bandeloos", Die Naweek, 14 Okcober 1943. [Text reference: Petersen, 'Bandeloos'] —. 1943. "Rotjies", Die Naweek, 25 March 1943. [Text reference: Petersen, 'Rotjies']
—. 1945. "In die hart van die stad", Die Naweek, 27 September 1945. [Text reference: Petersen, 'In die hart van die stad']

—. 1945. "Op Nuweland", Die Naweek, 16 August 1945. [Text reference: Petersen, 'Op Nuweland']

—. 1946. As die son ondergaan. Port Elizabeth/Cape Town: UnieVolkspers Bpk. [Text reference: Petersen, As die son ondergaan]

- Texts by Small, Adam: Small, Adam. 1964. "Kanna, kô hystoe". In Links, Tony and Wium van Zyl. 1993. Syferfonteine. Cape Town: Tafelberg [Text reference: Small, Koza, $\boldsymbol{K} \boldsymbol{K H}]$

—. 1964. "Klein Kytie". In Anon. 1964. Windroos. Johannesburg: Afrikaanse Pers-boekhandel. [Text reference: Small, $\boldsymbol{K K}]$

—. 1973 [1961]. Kitaar my

Kruis. Cape Town/Pretoria:

Hollandsch Afrikaansche Uitgevers Maatschappij. [Text reference: Small, KMK]

-. 1973. Oos wes tuis bes Distrik Ses. Cape Town/ Pretoria: Human \& Rousseau. [Text reference: Small, DS]

—. 1975 [1965]. Kanna hy kô hystoe. Cape town: Tafelberg. [Text reference: Small, $\boldsymbol{K H} \boldsymbol{K H}$ ]

—. 1981 [1963]. Sê sjibbolet. Johannesburg/ Cape Town: Perskor. [Text reference: Small, $\boldsymbol{S S}$ ]

- Texts by Snyders, Peter:

Snyders, Peter. 1983. Political Joke. Johannesburg/Cape Town: Perskor. [Text reference: Snyders, Political Joke]

—. "Nursie" (unpublished short prose text) [Text reference: Snyders, Nursie] 
—. "Onopgeloste karma" (unpublished short prose text) [Text reference: Snyders, Onopgeloste karma ]

—. "Voete op aarde" (unpublished short prose text) [Text reference: Snyders, Voete op aarde]

\section{Journalistic texts:}

- News reports published In

- Die Burger (1/4/2002) [Text reference: Die Burger]

- $\quad$ Rapport (5/11/2012; 23/3/2014) [Text reference: Rapport]

- Son $(11 / 3 / 2011 ; 15 / 7 / 2011$; 21/10/2011;16/2/2012; 7/8/2013; $18 / 2 / 2014 ; 3 / 3 / 2014 ; 6 / 6 / 2014$; 3/2/2015;19/4/2015;12/5/2015) [Text reference: Son]

- Volksblad (21/6/2012) [Text reference: Volksblad]

\section{Journalistic columns:}

- Various contributions to the following columns in the newspaper Son:

- 'Oom Sonnie' [Text reference: Son, 'Oom Sonnie']
- 'Antie Mona' [Text reference: Son, 'Antie Mona']

- "Ditjies en Datjies" [Text reference: Son, 'Ditjies en Datjies']

- Column texts in Kaaps by Peter Snyders published in

- Die Burger (6/2/2014) [Text reference: Die Burger]

- Die Burger Landelik (29/3/2001, 12/4/2001, 10/5/2001, 24/5/2001, 6/6/2001, 23/8/2001) [Text reference: Die Burger Landelik]

- Beeld PLUS (17/2/1998) [Text reference: Beeld PLUS]

- The column "Sypaadjies" in Rapport Weekliks, managed by Nathan Trantraal [Text reference: Rapport Weekliks]

- Some own recordings

Frank Hendricks is an emeritus professor in Linguistics in the Department of Afrikaans and Nederlands at the University of the Western Cape. fhendricks@uwc.ac.za 